\title{
Quasistatischer Normalstoß axialsymmetrischer Körper
}

Basierend auf den geschilderten kontaktmechanischen Grundlagen (teilweise in deren Interpretation durch die MDR) sind in diesem Kapitel die Lösungen des Normalstoßproblems in unterschiedlichen Fällen dargestellt. Betrachtet werden homogene und inhomogene elastische Medien mit und ohne Adhäsion sowie elasto-plastische und viskoelastische Materialien.

Da alle gewonnenen kontaktmechanischen Ergebnisse aus den Gleichgewichtsbedingungen eines elastischen Mediums hergeleitet wurden, ist klar, dass die Verwendung dieser Ergebnisse nur zulässig ist, falls die Kontaktkräfte zu jedem Zeitpunkt denen des statisch deformierten Mediums entsprechen. Diese (zusätzliche) grundlegende Annahme der Quasistatik wird im ersten Teil des Kapitels genauer erläutert.

Im Rahmen der Quasistatik ist die allgemeine Kollision verformbarer Kugeln, wie im zweiten und dritten Kapitel gezeigt, äquivalent zu dem Stoß einer starren Kugel auf einen deformierbaren Halbraum, da sowohl die dynamischen als auch die kontaktmechanischen Eigenschaften konsistent abgebildet werden. Für den zentrischen Normalstoß gilt das auch für beliebige axialsymmetrische Körper, da die Dynamik in diesem Fall elementar ist. Im Folgenden wird daher nur der Stoß eines starren Eindruckkörpers auf einen deformierbaren Halbraum betrachtet.

\subsection{Quasistatik}

Alle Prozesse während des Stoßes sollen quasistatisch verlaufen. Es ist unmittelbar klar, dass dafür die makroskopischen Geschwindigkeiten der zusammenstoßenden Körper klein gegenüber der charakteristischen Geschwindigkeit der Ausbreitung elastischer Wellen in den Körpern sein müssen. Aber wie klein? Hertz [1] selbst, der auf der Grundlage seiner Lösung des Hertzschen Kontaktproblems auch das entsprechende quasistatische Normalstoßproblem untersuchte, stellte dafür zwei konkretisierende Forderungen auf, eine 
schwächere und eine stärkere. Die schwächere Variante ist, dass die gesamte Stoßzeit $T_{S}$ sehr groß gegenüber der Zeit

$$
T_{\mathrm{el}}=\frac{2 a_{\mathrm{max}}}{v_{\mathrm{el}}}, \quad v_{\mathrm{el}}:=\sqrt{\frac{1}{\rho} \frac{2 G(1-v)}{1-2 v}},
$$

mit der Dichte $\rho$, ist, die elastische Kompressionswellen brauchen, um das maximale Kontaktgebiet während des Stoßes zu durchlaufen. Nach Eason [2] ist das ebenfalls die Zeit, nach der die normale Verschiebung im Zentrum einer plötzlich aufgebrachten, konstanten oder parabolischen Druckverteilung ihren statischen Wert erreicht. Das Verhältnis der beiden charakteristischen Zeiten ist von der Größenordnung

$$
\frac{T_{S}}{T_{\mathrm{el}}} \approx \frac{d_{\max }}{a_{\max }} \frac{v_{\mathrm{el}}}{v} .
$$

Das Verhältnis der Stoßgeschwindigkeit zur Ausbreitungsgeschwindigkeit elastischer Wellen muss also deutlich kleiner sein als die Oberflächengradienten im Kontaktgebiet (die nach der Halbraumnäherung selbst bereits klein sind). Eine noch stärkere Forderung ist die Annahme, dass sich im quasistatischen Grenzfall die an den Grenzen der Körper (mehrfach) reflektierten elastischen Wellen während des Stoßes im Kontaktgebiet überlagern, dass also die Stoßzeit sehr groß gegenüber der Zeit ist, die elastische Kompressionswellen brauchen, um die gesamten zusammenstoßenden Körper zu durchlaufen. Love [3, S. 195 ff.] führte aus, dass im Fall elastischer kollidierender Kugeln dafür

$$
\left(\frac{v}{v_{\mathrm{el}}}\right)^{1 / 5} \ll 1
$$

sein muss. Für den Stoß einer starren Kugel auf einen elastischen Halbraum (der, wie gezeigt, aus Sicht der makroskopischen Dynamik und der statischen Kontaktmechanik dem Stoß zweier elastischer Kugeln äquivalent ist) ist diese Forderung gar nicht erfüllbar, da es keine Grenze gibt, an der die elastischen Wellen reflektiert werden könnten. Das ist allerdings auch nicht notwendig für die quasistatische Behandlung, da von Hunter [4] gezeigt wurde, dass diese stärkere Forderung unnötig restriktiv ist. Es reicht aus, wenn die in Form elastischer Wellen abgestrahlte Energie (dies ist auch für die Bestimmung der Stoßzahlen die letztlich entscheidende Größe) klein gegenüber der vor dem Stoß in der Bewegung gespeicherten kinetischen Energie ist. Dazu muss laut Hunter lediglich

$$
\left(\frac{v}{\tilde{v}_{\mathrm{el}}}\right)^{3 / 5} \ll 1, \quad \tilde{v}_{\mathrm{el}}:=\sqrt{\frac{E}{\rho}},
$$

sein, was eine deutlich schwächere Forderung darstellt als Gl. (5.3). Für die Verwendbarkeit der nachfolgenden Ergebnisse soll die makroskopische charakteristische Geschwindigkeit 
$v$ vor dem Stoß also etwa drei Größenordnungen kleiner sein als die charakteristische Ausbreitungsgeschwindigkeit elastischer Wellen.

Eine sehr gute Übersicht zu voll-dynamischen Prozessen bei Kollisionen bietet die Monografie von Goldsmith [5].

\subsection{Elastischer Normalstoß ohne Adhäsion}

\subsubsection{Homogene Medien}

Im quasistatischen Grenzfall können für die Kontaktkräfte ihre statischen Werte angenommen werden. Mit den Gl. (2.34), (3.35) und (3.36) ist damit die Bewegungsgleichung für den Normalstoß einer starren Kugel mit der Masse $\tilde{m}$ und dem Radius $\tilde{R}$ auf einen homogenen elastischen Halbraum mit dem effektiven Elastizitätsmodul $\tilde{E}$ durch

$$
\tilde{m} \ddot{d}=-\frac{4}{3} \tilde{E} \sqrt{\tilde{R} d^{3}}
$$

gegeben. Die Lösung dieser Gleichung geht auf Hertz [1], bzw. Deresiewicz [6] zurück. Integration von (5.5) über die Indentierungstiefe unter Verwendung der Anfangsbedingungen

$$
d(t=0)=0, \quad \dot{d}(t=0)=v_{0}
$$

liefert die Gleichung der Erhaltung der mechanischen Gesamtenergie,

$$
\frac{\tilde{m}}{2} v_{0}^{2}=\frac{\tilde{m}}{2} \dot{d}^{2}+\frac{8}{15} \tilde{E} \sqrt{\tilde{R} d^{5}}
$$

aus der sich ohne größere Schwierigkeiten die maximale Eindrucktiefe,

$$
d_{\max }=\left(\frac{15 \tilde{m} v_{0}^{2}}{16 \tilde{E} \sqrt{\tilde{R}}}\right)^{2 / 5}
$$

und die dazugehörige maximale Normaldruckkraft,

$$
F_{N, \max }=\frac{4}{3} \tilde{E} \sqrt{\tilde{R} d_{\max }^{3}}=\left(\frac{125}{36} \tilde{m}^{3} v_{0}^{6} \tilde{R} \tilde{E}^{2}\right)^{1 / 5},
$$

bestimmen lassen. Mithilfe der Trennung der Variablen,

$$
\mathrm{d} t=\frac{1}{v_{0}} \frac{\mathrm{d} d}{\sqrt{1-\left(\frac{d}{d_{\max }}\right)^{5 / 2}}}
$$


erhält man außerdem die Inverse der Bahn ${ }^{1}$,

$$
t(d)=\frac{2 d_{\max }}{5 v_{0}} \mathrm{~B}\left(\xi ; \frac{2}{5}, \frac{1}{2}\right), \quad \xi:=\left(\frac{d}{d_{\max }}\right)^{5 / 2},
$$

und damit die gesamte Stoßdauer,

$$
T_{S}=\frac{4 d_{\max }}{5 v_{0}} \mathrm{~B}\left(1 ; \frac{2}{5}, \frac{1}{2}\right)
$$

B $(\cdot ; \cdot, \cdot)$ bezeichnet dabei die in Gl. (9.42) im Anhang definierte unvollständige Beta-Funktion. Mit der maximalen Eindrucktiefe ist nach Gl. (3.37) auch der Maximaldruck im Kontakt während des Stoßes als

$$
p_{\max }=\frac{2 \tilde{E}}{\pi} \sqrt{\frac{d_{\max }}{\tilde{R}}}=\frac{2}{\pi}\left(\frac{15 \tilde{E}^{4} \tilde{m} v_{0}^{2}}{16 \tilde{R}^{3}}\right)^{1 / 5} \approx \tilde{E}\left(\frac{v_{0}}{\tilde{v}_{\mathrm{el}}}\right)^{2 / 5}
$$

bekannt. Für die meisten metallischen Werkstoffe ist die Fließgrenze zwei bis drei Größenordnungen kleiner als der Elastizitätsmodul, die Stoßgeschwindigkeit muss dann etwa 5 bis 7 Größenordnungen unterhalb der Ausbreitungsgeschwindigkeit elastischer Wellen liegen, damit es zu keiner nennenswerten plastischen Deformation während des Stoßes kommt; die Annahme der Elastizität des Stoßes liefert daher eine stärkere Beschränkung für die makroskopische Geschwindigkeit vor dem Stoß als die Bedingung (5.4) für die Quasistatik, d.h. mögliche Energieverluste während des Stoßes sind eher auf plastische Deformationen zurückzuführen als auf die Energieabstrahlung in Form elastischer Wellen.

Für die Bahnkurve wurde von Hunter [4] die einfache Näherung

$$
d(t) \approx d_{\max } \sin \left(\frac{\pi t}{T_{S}}\right)
$$

angegeben. Die Stoßzahl für den elastischen Normalstoß ist im quasistatischen Grenzfall trivialerweise Eins.

Völlig analog erhält man für einen Indenter mit einem Profil in der Form eines Potenzgesetzes - siehe Gl. (3.38) - mithilfe der Lösung des Kontaktproblems aus den Gl. (3.39) und (3.40) die Gleichung der Energieerhaltung,

$$
\frac{\tilde{m}}{2} v_{0}^{2}=\frac{\tilde{m}}{2} \dot{d}^{2}+\frac{2 n^{2}}{(2 n+1)(n+1)} \frac{\tilde{E}}{[\beta(n) A]^{1 / n}} d^{\frac{2 n+1}{n}},
$$

und damit die, zuerst von Graham [7] publizierte, Stoßlösung

\footnotetext{
${ }^{1}$ während der Eindringphase; für den Rückprall muss entsprechend gespiegelt werden
} 


$$
\begin{aligned}
d_{\max } & =\left\{\frac{[\beta(n) A]^{1 / n}(2 n+1)(n+1) \tilde{m} v_{0}^{2}}{4 n^{2} \tilde{E}}\right\}^{\frac{n}{2 n+1}}, \\
t(d) & =\frac{n}{2 n+1} \frac{d_{\max }}{v_{0}} \mathrm{~B}\left(\xi ; \frac{n}{2 n+1}, \frac{1}{2}\right), \quad \xi:=\left(\frac{d}{d_{\max }}\right)^{\frac{2 n+1}{n}}, \\
T_{S} & =\frac{2 n}{2 n+1} \frac{d_{\max }}{v_{0}} \mathrm{~B}\left(1 ; \frac{n}{2 n+1}, \frac{1}{2}\right) .
\end{aligned}
$$

Die Näherung (5.14) ist die exakte Lösung für $n \rightarrow \infty$ (also für einen flachen zylindrischen Stempel), denn ${ }^{2}$

$$
\mathrm{B}\left(z^{2} ; \frac{1}{2}, \frac{1}{2}\right) \equiv 2 \arcsin (z)
$$

Abb.5.1 zeigt die normierten Trajektorien für einen konischen $(n=1)$ und parabolischen ( $n=2$ ) Indenter sowie den Fall $n=5$. Die Näherung (5.14) ist als dünne Linie ebenfalls hinzugefügt. Wie man sieht, unterscheiden sich die normierten Trajektorien für verschiedene Exponenten $n$ nur geringfügig von dem harmonischen Grenzfall. Das Profil der kollidierenden Körper hat also in der Regel nur einen geringen Einfluss auf das elastische Normalstoßproblem ohne Adhäsion.

Abb.5.1 Trajektorien während der Kompressionsphase des Stoßes eines starren Indenters mit einem Profil in der Form eines Potenzgesetzes auf einen elastischen Halbraum für verschiedene Exponenten $n$ des Potenzgesetzes. Die dünne durchgezogene Linie beschreibt die Näherung (5.14)

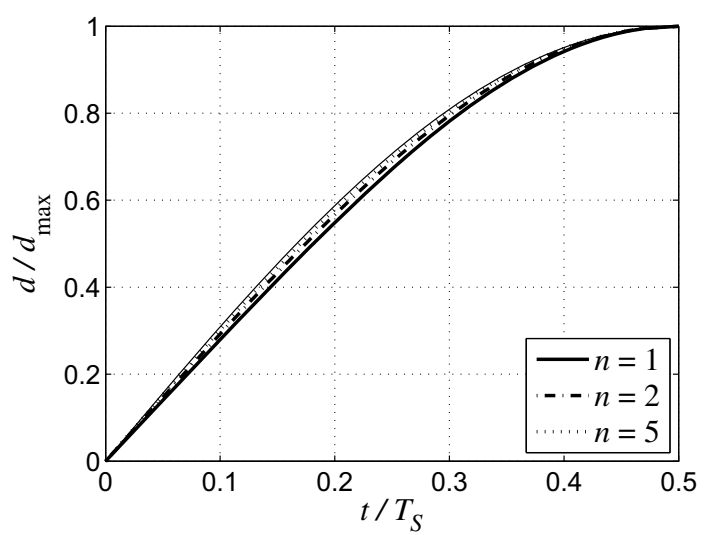

${ }^{2}$ Dieser Grenzfall ist sofort klar, da der Normalkontakt mit einem flachen zylindrischen Stempel eine konstante Steifigkeit besitzt, die Stoßlösung also eine harmonische Funktion sein muss. 


\subsubsection{Funktionale Gradientenmedien}

Völlig analog zum homogenen Fall kann auch für Medien mit einer elastischen Gradierung in der Form eines Potenzgesetzes das Normalstoßproblem im quasistatischen Grenzfall analytisch gelöst werden [8]. Für den Stoß von Kugeln nimmt die Energieerhaltung in diesem Fall die Form

$$
\frac{\tilde{m}}{2} v_{0}^{2}=\frac{\tilde{m}}{2} \dot{d}^{2}+\frac{8 c_{N}}{(3+k)(5+k)} \sqrt{(1+k)^{k-1} \tilde{R}^{1+k} d^{5+k}}
$$

an, aus der sich wiederum die maximale Eindrucktiefe, die Inverse der Bahn und die gesamte Stoßdauer zu

$$
\begin{aligned}
d_{\max } & =\left[\frac{(3+k)(5+k) \tilde{m} v_{0}^{2}}{16 c_{N} \sqrt{(1+k)^{k-1} \tilde{R}^{1+k}}}\right]^{\frac{2}{5+k}}, \\
t(d) & =\frac{2 d_{\max }}{(5+k) v_{0}} \mathrm{~B}\left(\xi ; \frac{2}{5+k}, \frac{1}{2}\right), \quad \xi:=\left(\frac{d}{d_{\max }}\right)^{\frac{5+k}{2}}, \\
T_{S} & =\frac{4 d_{\max }}{(5+k) v_{0}} \mathrm{~B}\left(1 ; \frac{2}{5+k}, \frac{1}{2}\right)
\end{aligned}
$$

bestimmen lassen. Der maximale Druck im Kontakt,

$$
p_{\max }=\frac{2}{\pi} c_{N}^{\frac{4}{5+k}} \tilde{R}^{\frac{k-3}{5+k}}(1+k)^{\frac{k-7}{5+k}}\left[\frac{\tilde{m} v_{0}^{2}(3+k)(5+k)}{16}\right]^{\frac{1+k}{5+k}}
$$

kann auf den entsprechenden Wert im homogenen Fall normiert werden. Man erhält:

$$
\frac{p_{\max }}{p_{\max }^{k=0}}=\left(\frac{c_{N}}{\tilde{E}}\right)^{\frac{4}{5+k}}\left(a_{\max }^{k=0}\right)^{\frac{4 k}{5+k}}(1+k)^{\frac{k-7}{5+k}}\left[\frac{(3+k)(5+k)}{15}\right]^{\frac{1+k}{5+k}}
$$

mit dem maximalen Kontaktradius während des Stoßes im homogenen Fall,

$$
a_{\max }^{k=0}=\left(\frac{15 \tilde{m} v_{0}^{2} \tilde{R}^{2}}{16 \tilde{E}}\right)^{1 / 5}
$$

Gl. (5.25) kann man auch in der logarithmischen Form

$$
\log \left(\frac{p_{\max }}{p_{\max }^{k=0}}\right)=\log \left\{(1+k)^{\frac{k-7}{5+k}}\left[\frac{(3+k)(5+k)}{15}\right]^{\frac{1+k}{5+k}}\right\}+\frac{4}{5+k} \log \left[\frac{\left(a_{\max }^{k=0}\right)^{k} c_{N}}{\tilde{E}}\right]
$$


schreiben. Da $c_{N}$ mit $z_{0}^{-k}$ skaliert, wobei $z_{0}$ die charakteristische Tiefe der Gradierung darstellt, erkennt man, dass für positive Exponenten $k$, also weiche Oberflächen, die Gradierung tief im Vergleich mit dem charakteristischen Kontaktradius sein muss um kleinere Spannungen zu erreichen; im umgekehrten Fall harter Oberflächen, sprich negativer Exponenten $k$, muss die Gradierung dagegen möglichst dünn sein, um die Spannungen zu reduzieren. In diesem Fall trägt der weiche Kern des Mediums einen großen Teil der Kontaktlast.

Das Stoßproblem kann auch für einen allgemeinen rotationssymmetrischen Indenter mit einem Profil in der Form eines Potenzgesetzes gelöst werden, da die Lösung des Kontaktproblems bekannt ist. Es ergeben sich die Beziehungen

$$
\begin{aligned}
d_{\max } & =\left\{\frac{[\beta(n, k) A]^{\frac{1+k}{n}}(2 n+k+1)(n+k+1)(1+k) \tilde{m} v_{0}^{2}}{4 n^{2} c_{N}}\right\}^{\frac{n}{2 n+k+1}}, \\
t(d) & =\frac{n}{2 n+k+1} \frac{d_{\max }}{v_{0}} \mathrm{~B}\left(\xi ; \frac{n}{2 n+k+1}, \frac{1}{2}\right), \quad \xi:=\left(\frac{d}{d_{\max }}\right)^{\frac{2 n+k+1}{n}}, \\
T_{S} & =\frac{2 n}{2 n+k+1} \frac{d_{\max }}{v_{0}} \mathrm{~B}\left(1 ; \frac{n}{2 n+k+1}, \frac{1}{2}\right) .
\end{aligned}
$$

Man erkennt, dass die normierte Gleichung der Bahnkurve

$$
\hat{t}:=\frac{v_{0}}{d_{\max }} t=\hat{t}(\hat{d}), \quad \hat{d}:=\frac{d}{d_{\max }},
$$

durch die homogene Lösung (5.17) gegeben ist, wenn in dieser der „korrigierte“ Exponent des Potenzprofils - siehe Gl.(3.241) -

$$
\tilde{n}=\frac{n}{1+k}
$$

verwendet wird. Das Normalstoßproblem eines Indenters mit dem Exponent $n$ auf einen elastischen Halbraum mit einer Gradierung in der Form eines Potenzgesetzes mit dem Exponenten $k$ kann also in entsprechend normierten Größen auf das jeweilige homogene Problem mit dem in Gl.(5.32) gegebenen angepassten Exponenten $\tilde{n}$ des Indenterprofils zurückgeführt werden. Das liegt daran, dass beide Probleme den gleichen funktionalen Zusammenhang für die Kontaktsteifigkeit in Abhängigkeit der Eindrucktiefe aufweisen.

Es ist klar, dass die Bahnkurve für $k \rightarrow-1$ grundsätzlich (also unabhängig von $n$ ) gegen die harmonische Lösung (5.14) strebt, da das ,,äquivalente“ homogene Problem durch $\tilde{n} \rightarrow \infty$, also den zylindrischen Flachstempel, gegeben ist $^{3}$.

\footnotetext{
${ }^{3}$ Dies kann man auch dadurch verstehen, dass die inkrementelle Kontaktsteifigkeit nach Gl. (3.226) für $k \rightarrow-1$ nicht von der Kontaktkonfiguration abhängt und sich der Kontakt damit in diesem Grenzfall grundsätzlich wie eine lineare Feder verhält.
} 


\subsection{Elastischer Normalstoß mit Adhäsion}

Dieses Unterkapitel widmet sich dem elastischen Normalstoß unter Berücksichtigung der Adhäsion. Da diese die zur Erzeugung plastischer Deformationen nötige äußere Last reduziert, spielen plastische Verformungen in adhäsiven Kollisionen eine noch größere Rolle als in nicht-adhäsiven. Dieser Aspekt wird im Unterkapitel 5.6 diskutiert.

\subsubsection{Homogene Medien mit JKR-Adhäsion}

Die Bewegungsgleichung für den JKR-adhäsiven Normalstoß von elastisch homogenen Kugeln ist im quasistatischen Grenzfall wegen der Gl.(2.34), (3.55) und (3.56) durch das System

$$
\begin{aligned}
\tilde{m} \ddot{d} & =-\frac{4 \tilde{E} a^{3}}{3 \tilde{R}}+\sqrt{8 \pi a^{3} \tilde{E} \Delta \gamma}, \\
d & =\frac{a^{2}}{\tilde{R}}-\sqrt{\frac{2 \pi a \Delta \gamma}{\tilde{E}}}
\end{aligned}
$$

gegeben. Dieses ließe sich theoretisch in eine explizite Gleichung für den Kontaktradius $a$ überführen, das wäre aber äußerst unhandlich. Die Bewegungsgleichung lässt sich auch nicht, wie im nicht-adhäsiven Fall, analytisch lösen. Allerdings kann man den maximalen Kontaktradius (und damit die gesamte Kontaktkonfiguration am Umkehrpunkt) und den Energieverlust während des Stoßes analytisch bestimmen, wie von Johnson und Pollock [9] sowie Thornton und Ning [10] demonstriert wurde.

Im Kapitel zu den kontaktmechanischen Grundlagen wurde dargelegt, dass sich im Moment der ersten Berührung durch die Wirkung der ab diesem Moment ,spürbaren“ Adhäsion spontan ein Kontaktgebiet mit dem Radius $a_{0}$ ausbildet, siehe Gl. (3.57). Der maximale Kontaktradius während des Stoßes ergibt sich daher aus der Lösung der Gleichung der einfachen Energiebilanz:

$$
\frac{\tilde{m}}{2} v_{0}^{2}=-\int_{a_{0}}^{a_{\max }} F_{z} \frac{\mathrm{d} d}{\mathrm{~d} a} \mathrm{~d} a=\frac{8 \tilde{E}}{15 \tilde{R}^{2}} a_{\max }^{5}-\frac{8}{3} \sqrt{\frac{\pi \Delta \gamma \tilde{E}}{2 \tilde{R}^{2}} a_{\max }^{7}}+\pi \Delta \gamma a_{\max }^{2}+\frac{3}{5}\left[\frac{4(\pi \Delta \gamma)^{5} \tilde{R}^{4}}{\tilde{E}^{2}}\right]^{1 / 3} .
$$

Während des Stoßes sind alle Deformationen elastisch, der Zustand $d=0$ wird also mit der betragsmäßig gleichen Relativgeschwindigkeit erreicht, wie beim Aufprall. Allerdings wird der Kontakt erst aufgelöst, wenn er bei $d_{c}^{\mathrm{WS}}<0$ - siehe Gl.(3.60) - seine Stabilität 
verliert $^{4}$, wodurch zusätzliche Arbeit gegen die Wirkung der Adhäsion verrichtet werden muss. Der adhäsive Verlust an kinetischer Energie ist durch

$$
\Delta U_{\text {kin }}=\int_{a_{0}}^{a_{c}^{\text {Ws }}} F_{z} \frac{\mathrm{d} d}{\mathrm{~d} a} \mathrm{~d} a=-\frac{1}{10}(1+\sqrt[3]{864})\left[\frac{\tilde{R}^{4}(\pi \Delta \gamma)^{5}}{\tilde{E}^{2}}\right]^{1 / 3} \approx-0,613\left[\frac{81 \tilde{R}^{4}(\pi \Delta \gamma)^{5}}{16 \tilde{E}^{2}}\right]^{1 / 3}
$$

gegeben, woraus sich wegen

$$
\epsilon_{z}=\sqrt{1+\frac{2 \Delta U_{\text {kin }}}{\tilde{m} v_{0}^{2}}}, \quad\left|\Delta U_{\text {kin }}\right| \leq \frac{m v_{0}^{2}}{2},
$$

auch sofort die normale Stoßzahl ergibt. Der adhäsive Normalstoß hat also die interessante Eigenschaft, dass der Energie-Verlust während der Kollision nicht von der Stoßgeschwindigkeit abhängt. Die Stoßzahl wird daher für sinkende Kollisionsgeschwindigkeiten immer kleiner. Ist die Geschwindigkeit $v_{0}$ kleiner als die kritische Geschwindigkeit

$$
v_{c}:=\sqrt{\frac{2}{\tilde{m}} 0,613\left[\frac{81 \tilde{R}^{4}(\pi \Delta \gamma)^{5}}{16 \tilde{E}^{2}}\right]^{1 / 3}},
$$

bleiben die Kugeln aneinander kleben und lösen sich nicht wieder voneinander. Die Stoßzahl ist dann gleich Null.

Auf die gleiche Art und Weise erhält man für einen allgemeinen axialsymmetrischen Indenter mit einem Profil in der Form eines Potenzgesetzes mithilfe der Lösung des adhäsiven Normalkontaktproblems aus den Gl.(3.65) und (3.66) den adhäsiven Verlust an kinetischer Energie:

$$
\Delta U_{\mathrm{kin}}=\frac{2-4 n}{2 n^{2}+3 n+1}\left[n+(n+1)(2 n)^{\frac{4}{2 n-1}}\right]\left(\frac{\pi \Delta \gamma}{2}\right)^{\frac{2 n+1}{2 n-1}}\left(\frac{1}{\tilde{E} n^{2} \beta^{2}(n) A^{2}}\right)^{\frac{2}{2 n-1}} .
$$

Dabei muss beachtet werden, dass diese Beziehung nur für $n>1 / 2$ verwendet werden kann und $\Delta U_{\text {kin }}$ daher auch immer negativ ist.

\footnotetext{
${ }^{4}$ Für das Stoßproblem ist $d$ der durch die Bewegung der Kugel vorgegebene Kontrollparameter, das Kontaktproblem muss daher unter weggesteuerten Bedingungen gelöst werden.
} 


\subsubsection{Homogene Medien mit Adhäsion nach Maugis (parabolischer Kontakt)}

Mit der Lösung des Kontaktproblems aus Abschn.3.3.3 kann man auch das NormalstoßProblem mit Adhäsion in der Beschreibung von Maugis behandeln [11]. Es wird dabei im vorliegenden Abschnitt von den gleichen normierten Größen Gebrauch gemacht wie bei der Schilderung der Lösung des Kontaktproblems, alle verwendeten Notationen können daher im dritten Kapitel nachgeschlagen werden.

Der Stoß beginnt, wenn der Abstand zwischen dem Halbraum und dem Punkt des ersten Kontaktes auf dem Eindruckkörper kleiner ist als die Reichweite der Adhäsion, und damit bei einer normierten Eindrucktiefe von

$$
\hat{d}_{0}=-\frac{1}{\Lambda}
$$

Wenn der Stoß ohne direkten Kontakt beginnt, bildet sich nach Gl.(3.83) spontan eine adhäsive Zone mit dem Radius

$$
\hat{b}_{0}=\frac{64}{3 \pi^{2}} \Lambda
$$

Nach der Ungleichung (3.81) sind solche Konfigurationen möglich, falls

$$
\frac{3 \pi^{3}}{2^{9} \Lambda^{3}} \geq 2 \Rightarrow \Lambda \leq 0,45
$$

Es ergeben sich also drei verschiedene Fälle: Für $\Lambda \leq 0,45$ beginnt und endet der Stoß ohne direkten Kontakt. Für 0,45 $<\Lambda \leq 0,64$ - siehe Gl. (3.89) - beginnt der Stoß mit direktem Kontakt und endet ohne ihn. Für $\Lambda>0,64$ beginnt und endet der Stoß mit direktem Kontakt.

Der Verlust an kinetischer Energie während des Stoßes, aus dem man mit Gl. (5.37) auch die Stoßzahl bestimmen kann, ergibt sich zu

$$
\Delta U_{\text {kin }}=\int_{-h_{1}}^{d_{c}^{\mathrm{WS}}} F_{z} \mathrm{~d} d=\frac{15 \sqrt[3]{3}}{4(1+\sqrt[3]{864})}\left|\Delta U_{\mathrm{kin}}^{\mathrm{JKR}}\right| \int_{-1 / \Lambda}^{\hat{d}_{c}^{\mathrm{WS}}} \hat{F}_{z} \mathrm{~d} \hat{d},
$$

mit dem in Gl. (5.36) angegebenen Verlust im JKR-Grenzfall. Zur Bestimmung des verbleibenden Integrals muss man die beschriebenen drei Fälle unterscheiden.

\section{Fall 1: Stoß beginnt und endet ohne direkten Kontakt}

Im Fall sehr kleiner Werte des Tabor-Parameters, für die der Stoß ohne direkten Kontakt beginnt und endet, kann man das Integral ohne Schwierigkeiten analytisch auswerten: 


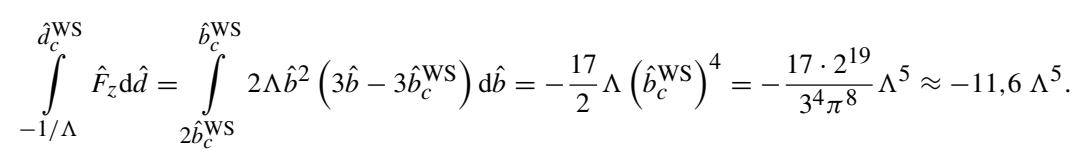

\section{Fall 2: Stoß beginnt mit direktem Kontakt, endet ohne ihn}

Falls der Stoß mit direktem Kontakt beginnt, aber ohne ihn endet, zerfällt das Integral in zwei Teile,

$$
\int_{-1 / \Lambda}^{\hat{d}_{c}^{\mathrm{WS}}} \hat{F}_{z} \mathrm{~d} \hat{d}=\int_{-1 / \Lambda}^{\hat{d}_{a 0}} \hat{F}_{z} \mathrm{~d} \hat{d}+\int_{\hat{d}_{a 0}}^{\hat{d}_{c}^{\mathrm{WS}}} \hat{F}_{z} \mathrm{~d} \hat{d}
$$

mit der Eindrucktiefe $\hat{d}_{a 0}$ aus Gl. (3.85), bei der der direkte Kontakt endet. Den Beitrag ohne direkten Kontakt kann man wiederum analytisch bestimmen:

$$
\int_{\hat{d}_{a 0}}^{\hat{d}_{c}^{\mathrm{WS}}} \hat{F}_{z} \mathrm{~d} \hat{d}=-\frac{2^{19} \Lambda^{5}}{3^{4} \pi^{8}}\left(3 K^{4}-4 K^{3}+1\right), \quad K:=1-\frac{\pi}{2}+\sqrt{\left(1-\frac{\pi}{2}\right)^{2}+\frac{3 \pi^{4}}{2^{9} \Lambda^{3}}} .
$$

Den Beitrag aus der Phase mit direktem Kontakt muss man numerisch ermitteln. Dazu wird mithilfe der Gl. (3.76) bis (3.78) die Normalkraft als Funktion der Eindrucktiefe bestimmt und anschließend numerisch integriert.

\section{Fall 3: Stoß beginnt und endet mit direktem Kontakt}

Für den Fall großer Werte des Tabor-Parameters muss man das gesamte Integral auf dem oben beschriebenen Weg numerisch bestimmen.

\section{Überschätzung des Hystere-Verlustes durch die JKR-Theorie}

Der so bestimmte adhäsive Energieverlust hängt, normiert auf den Wert im JKR-Grenzfall, nur von dem Tabor-Parameter ab und ist in Abb. 5.2 dargestellt. Offensichtlich ist der Verlust für einen endlichen Wert des Tabor-Parameters immer kleiner als im JKR-Grenzfall. Auch für $\Lambda=4$, dies entspricht einem ,ursprünglichen“ Tabor-Parameter von $\lambda_{T} \approx 5$, was im Allgemeinen bereits als ausreichend gute Näherung des JKR-Falles betrachtet wird, überschätzt das JKR-Ergebnis den Energieverlust um mehr als 10\%. Ciavarella et al. [12] gaben an, dass bei der Verwendung des Lennard-Jones-Potentials und der dazugehörigen adhäsiven Spannung (3.47) der Hysterese-Verlust bei $\lambda_{T}=5$ sogar nur knapp $50 \%$ des JKR-Ergebnisses beträgt. Dies liegt daran, dass durch die mit der Entfernung zwar gegen Null konvergierende aber niemals gänzlich verschwindende adhäsive Spannung der instabile Sprung in den Kontakt deutlich früher erfolgt, als durch die JKR- oder auch die MaugisTheorie vorhergesagt [13]. 
Abb. 5.2 Adhäsiver Verlust an kinetischer Energie für den Normalstoß von Kugeln mit Adhäsion nach Maugis, normiert auf den Wert im JKR-Grenzfall als Funktion des Tabor-Parameters $\Lambda$. Die durchgezogenen vertikalen Linien bezeichnen die Bereichsgrenzen der im Text beschriebenen Fälle

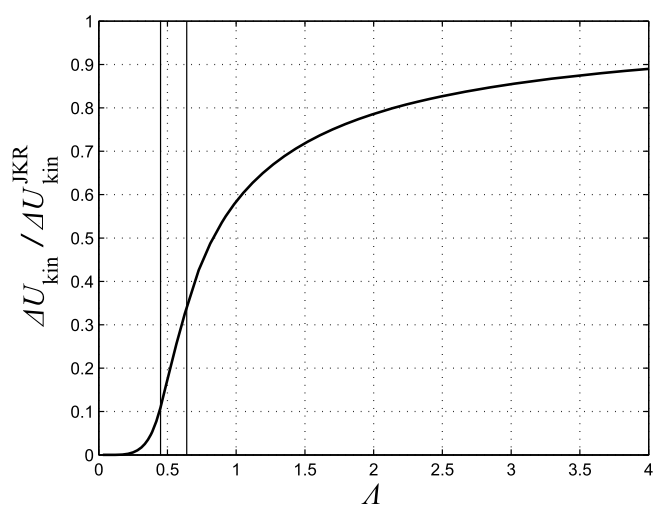

\subsubsection{Funktionale Gradientenmedien mit JKR-Adhäsion}

Völlig analog zum homogenen Fall kann man auch für Gradientenmedien mit einer Gradierung in der Form eines Potenzgesetzes, basierend auf der bekannten Kontaktlösung, den JKR-adhäsiven Normalstoß behandeln [14]. Die Bewegungsgleichung für den parabolischen Fall ist mit der Lösung des entsprechenden Kontaktproblems aus den Gl. (3.245) und (3.246) implizit durch die Zusammenhänge

$$
\begin{aligned}
\tilde{m} \ddot{d} & =-\frac{4 c_{N} a^{3+k}}{(1+k)^{2}(3+k) \tilde{R}}+\frac{2}{1+k} \sqrt{2 \pi c_{N} \Delta \gamma a^{3+k}}, \\
d & =\frac{a^{2}}{(1+k) \tilde{R}}-\sqrt{\frac{2 \pi a^{1-k} \Delta \gamma}{c_{N}}}
\end{aligned}
$$

gegeben. Der maximale Kontaktradius während des Stoßes ergibt sich als Lösung der Gleichung

$$
\frac{\tilde{m}}{2} v_{0}^{2}=\frac{8 c_{N} a_{\max }^{5+k}}{(1+k)^{3}(3+k)(5+k) \tilde{R}^{2}}-\frac{4}{(1+k)^{2}(3+k) \tilde{R}} \sqrt{2 \pi \Delta \gamma c_{N} a_{\max }^{7+k}}+\frac{1-k}{1+k} \pi \Delta \gamma a_{\max }^{2}+\Delta U_{0}
$$

mit

$$
\Delta U_{0}:=\frac{3+k}{5+k}\left[\frac{4(\pi \Delta \gamma)^{5+k} \tilde{R}^{4}(1+k)^{4}}{c_{N}^{2}}\right]^{\frac{1}{3+k}}
$$

Der adhäsive Verlust an kinetischer Energie während des Stoßes beträgt

$$
\Delta U_{\text {kin }}=-\Delta U_{0}\left[1+2^{-\frac{8}{3+k}} \frac{2+k}{(1+k)(3+k)}(1-k)^{\frac{7+k}{3+k}}\right] .
$$


Normiert man das auf das Ergebnis im homogenen Fall ergibt sich

$$
\frac{\Delta U_{\text {kin }}}{\Delta U_{\text {kin }}^{k=0}}=\frac{10 \cdot 2^{2 / 3}(3+k)(1+k)^{\frac{4}{3+k}}}{(5+k)(1+\sqrt[3]{864})}\left[\frac{\tilde{E}_{0}}{c_{N}\left(a_{0}^{k=0}\right)^{k}}\right]^{\frac{2}{3+k}}\left[1+2^{-\frac{8}{3+k}} \frac{2+k}{(1+k)(3+k)}(1-k)^{\frac{7+k}{3+k}}\right],
$$

was in logarithmischen Ausdrücken als

$$
\log \left(\frac{\Delta U_{\text {kin }}}{\Delta U_{\text {kin }}^{k=0}}\right)=C_{1}(k)+\frac{2}{3+k} \log \left[\frac{\tilde{E}_{0}}{c_{N}\left(a_{0}^{k=0}\right)^{k}}\right]
$$

geschrieben werden kann. Man erkennt, dass der Verlust jeweils für weiche Oberflächen $(k>0)$ mit flacherer Gradierung und für harte Oberflächen $(k<0)$ mit tieferer Gradierung kleiner wird. Ein kleinerer adhäsiver Energieverlust geht dabei wegen Gl. (5.27) allerdings mit höheren maximalen Kontaktspannungen einher.

\subsection{Viskoelastischer Normalstoß ohne Adhäsion}

In diesem Unterkapitel wird der viskoelastische Normalstoß für unterschiedliche linearviskoelastische Medien untersucht. Dabei finden die im dritten Kapitel eingeführten klassischen viskoelastischen Materialmodelle Anwendung. An gegebener Stelle werden die theoretischen Vorhersagen mit experimentellen Ergebnissen verglichen.

Da viskoelastische Medien häufig weich aber fest sind, gewinnt bei stoßartigen Belastungen dieser Materialien die Wellenausbreitung an Bedeutung. Diese wird trotzdem weiterhin vernachlässigt, d.h. es werden auch im Folgenden nur quasistatische Prozesse untersucht.

\subsubsection{Inkompressibles Kelvin-Voigt-Medium}

Man betrachte zunächst das Kelvin-Voigt-Medium. Dies stellt den einfachsten und, wie sich später ergibt, wichtigsten Fall dar. Im Folgenden werden der Flachstempelkontakt und der parabolische Kontakt genauer untersucht, die gezeigten Methoden kann man aber für beliebige konvexe, axialsymmetrische Eindruckkörper verwenden.

\section{Flachstempel-Kontakt}

Der gerade Normalstoß eines starren flachen zylindrischen Stempels mit dem Radius $a$ und der Masse $\tilde{m}$ auf einen inkompressiblen viskoelastischen Halbraum, der sich als linearer 
Kelvin-Voigt-Körper mit dem Schubmodul $G$ und der Scherviskosität $\eta$ modellieren lässt, wurde von Butcher und Segalman [15], Schwager und Pöschel [16] und Argatov [17] gelöst ${ }^{5}$.

Die Bewegungsgleichung für die Eindrucktiefe $d$ durch den Stempel ist unter quasistatischen Bedingungen und mithilfe der in früheren Kapiteln hergeleiteten kontaktmechanischen Ergebnisse durch

$$
\tilde{m} \ddot{d}+8 \eta a \dot{d}+8 G a d=0
$$

gegeben. Es ist nützlich, die Kürzel

$$
D \omega_{0}:=\frac{4 \eta a}{\tilde{m}}, \quad \omega_{d}:=\omega_{0} \sqrt{1-D^{2}}, \quad \omega_{0}^{2}:=\frac{8 G a}{\tilde{m}}
$$

einzuführen. Vergleicht man dabei mit dem komplexen Modul des Kelvin-Voigt-Mediums aus Gl. (3.187) wird deutlich, dass das Dämpfungsmaß $D$ als

$$
2 D=\frac{\eta \omega_{0}}{G}=\frac{\operatorname{Im}\left[\hat{G}\left(\omega_{0}\right)\right]}{\operatorname{Re}\left[\hat{G}\left(\omega_{0}\right)\right]}
$$

d.h. als Verhältnis des Verlust- und Speichermoduls bei $\omega=\omega_{0}$, gedeutet werden kann. Diese Deutung ist, wie im weiteren Verlauf gezeigt werden wird, in dieser allgemeinen Form auch für andere viskoelastische Materialmodelle zumindest eine gute Näherung.

Die Lösung der Bewegungsgleichung (5.54) mit den Anfangsbedingungen (5.6) ist elementar. Man erhält im Fall schwacher Dämpfung (also für $D<1$ )

$$
d(t)=\frac{v_{0}}{\omega_{d}} \exp \left(-D \omega_{0} t\right) \sin \left(\omega_{d} t\right)
$$

Der Stoß endet, falls die Normalkraft verschwindet ${ }^{6}$. Dies resultiert in der Forderung

$$
\tan \left(\omega_{d} T_{S}\right)=\frac{2 D \sqrt{1-D^{2}}}{D^{2}-\left(1-D^{2}\right)}
$$

für die Stoßdauer $T_{S}$. Mithilfe des Additionstheorems

$$
\tan (2 x)=\frac{2 \tan x}{1-\tan ^{2} x}
$$

\footnotetext{
${ }^{5}$ In den genannten Publikationen wurden dabei teilweise etwas andere, aber kontaktmechanisch äquivalente Problemstellungen bearbeitet; da der Flachstempel-Kontakt linear ist, entspricht das Problem einfach einer Punktmasse, die mit einem linearen Kelvin-Voigt-Element gegen eine starre Wand stößt. ${ }^{6}$ Man beachte, dass in manchen Publikationen die elastische Bedingung $d=0$ verwendet wird, die allerdings zu unphysikalischen Zugkräften im Kontakt und damit zu deutlich kleineren Stoßzahlen führt.
} 
Abb. 5.3 Stoßzahl als Funktion des Dämpfungsmaßes $D$ für den Normalstoß eines starren Flachstempels auf ein Kelvin-Voigt-Medium

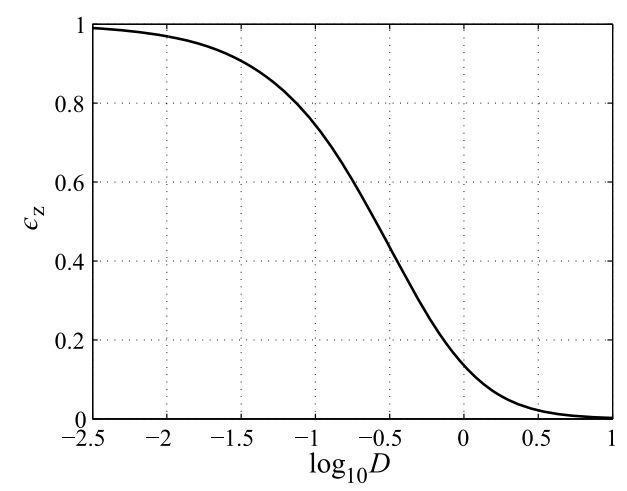

erhält man damit die Stoßdauer

$$
T_{S}=\frac{2}{\omega_{d}} \arctan \left(\frac{\sqrt{1-D^{2}}}{D}\right)
$$

und die Stoßzahl

$$
\epsilon_{z}=\exp \left(-D \omega_{0} T_{S}\right)=\exp \left[-\frac{2 D}{\sqrt{1-D^{2}}} \arctan \left(\frac{\sqrt{1-D^{2}}}{D}\right)\right], \quad D<1 .
$$

Der stark gedämpfte Fall $D>1$ wurde in den genannten Publikationen nicht untersucht. Man erhält aber völlig analog ${ }^{7}$

$$
\epsilon_{z}=\exp \left[-\frac{2 D}{\sqrt{D^{2}-1}} \operatorname{artanh}\left(\frac{\sqrt{D^{2}-1}}{D}\right)\right], \quad D>1,
$$

und im aperiodischen Grenzfall ganz einfach

$$
\epsilon_{z}=\exp (-2), \quad D=1 .
$$

Abb. 5.11 zeigt die Stoßzahl als Funktion des Dämpfungsmaßes $D$ in logarithmischer Skalierung.

\section{Parabolischer Kontakt}

Für den Normalstoß eines parabolischen Körpers mit dem Krümmungsradius $\tilde{R}$ ist die Lösung komplizierter, da sich der Kontaktradius $a$ während des Stoßes ändert. Er hat am Ende der Kompressionsphase ein einzelnes Maximum, daher müssen die Kompressionsund die Restitutionsphase getrennt voneinander betrachtet werden.

\footnotetext{
${ }^{7}$ Tatsächlich ist die ganze Herleitung für schwache Dämpfung auch für imaginäre Werte von $\omega_{d}$ korrekt.
} 
Während der Kompressionsphase ist die Lösung des Kontaktproblems, wegen der Gl.(3.202) und (3.203) sowie der Materialfunktion (3.186) des Kelvin-Voigt-Mediums, durch

$$
\begin{aligned}
F_{z}(t) & =-\frac{16 G}{3} \frac{a^{3}(t)}{\tilde{R}}-16 \eta \frac{a^{2}(t)}{\tilde{R}} \dot{a}, \\
\tilde{R} d(t) & =a^{2}(t)
\end{aligned}
$$

gegeben. Damit erhält man die Bewegungsgleichung

$$
\tilde{m} \ddot{d}+8 \eta \sqrt{\tilde{R}} d \dot{d}+\frac{16 G}{3} \sqrt{\tilde{R} d^{3}}=0 .
$$

Während der Restitutionsphase behält Gl.(5.64) zwar ihre Gültigkeit, doch Gl.(5.65) muss man wegen Gl.(3.206) durch

$$
\tilde{R} d(t)=a^{2}(t)-\tau \int_{t_{m}}^{t}\left[1-\exp \left(\frac{t^{\prime}-t}{\tau}\right)\right] \frac{\mathrm{d}^{2}}{\mathrm{~d} t^{\prime 2}} a^{2}\left(t^{\prime}\right) \mathrm{d} t^{\prime}
$$

mit der charakteristischen Kriechzeit $\tau:=\eta / G$, ersetzen.

Kuwabara und Kono [18] schlugen ein Modell für den Stoß vor, das später von einer Gruppe um Brilliantov und Pöschel in einer Serie von Publikationen [19-22] analysiert wurde, und bei dem die einfache Form der Bewegungsgleichung in der Kompressionsphase auch für die Restitutionsphase verwendet wird. Dies ist aus kontaktmechanischer Sicht leicht fehlerhaft $^{8}$ - die Normalkraft in der Restitutionsphase und damit die Stoßzahl werden systematisch unterschätzt - hat dafür aber den Vorteil, dass die Bewegungsgleichung während des gesamten Stoßes in expliziter Form bekannt und das entstehende Modell daher mathematisch sehr leicht lösbar ist. Es lässt sich deswegen einfach z. B. in molekulardynamische Simulationen granularer Medien integrieren.

Durch eine dimensionsfreie Formulierung des Bewegungsgleichungssystems kann man leicht zeigen, dass die Stoßzahl ausschließlich von dem Parameter

$$
\delta:=\eta\left(\frac{\tilde{R} v_{0}}{\tilde{m}^{2} G^{3}}\right)^{1 / 5}
$$

abhängt. Dieser gibt, bis auf einen konstanten Faktor der Größenordnung Eins, das Verhältnis zwischen den beiden inhärenten Zeitskalen des Problems wieder, der Kriechzeit $\tau$ des Kelvin-Voigt-Mediums und der elastischen Stoßdauer nach Gl. (5.12).

\footnotetext{
${ }^{8}$ Einen ähnlichen Fehler bei der Behandlung des viskoelastischen Problems - die Vernachlässigung der Tatsache, dass die Kompressions- und Restitutionsphase getrennt voneinander betrachtet werden müssen - begeht in seiner Arbeit auch Pao [23].
} 
In Abb. 5.4 ist die Stoßzahl als Funktion des einzigen Parameters $\delta$ gezeigt, einmal nach der kontaktmechanisch rigorosen Beschreibung (gelöst durch die MDR, siehe [24]) und einmal nach dem Modell von Kuwabara \& Kono. Man erkennt die systematische Unterschätzung der Stoßzahl durch das zweite Modell, der Unterschied ist allerdings sehr gering. Man muss dabei in Betracht ziehen, dass die Untersuchung des Stoßproblems mit dem KuwabaraKono-Modell einfacher und weniger rechenintensiv ist als die Lösung der aus kontaktmechanischer Sicht exakteren Gleichungen. Außerdem unterscheiden sich beide Verläufe nur sehr schwach von der in Abb. 5.3 gezeigten analytischen Lösung für den Flachstempel.

Der Energieverlust während des Stoßes ist durch

$$
\Delta U_{\text {kin }}=-8 \eta \int_{0}^{T_{S}} a \dot{d}^{2} \mathrm{~d} t
$$

bestimmt. Für sehr kleine Verluste kann man in erster Näherung annehmen, dass der Kontaktradius und die Eindrucktiefe als Funktionen der Zeit durch die jeweilige elastische Lösung siehe Gl.(5.11) - gegeben sind. Man erhält dann in erster Näherung für den Energieverlust

$$
\Delta U_{\mathrm{kin}} \approx-\frac{32}{5} \mathrm{~B}\left(\frac{3}{5}, \frac{3}{2}\right) \eta v_{0} \sqrt{\tilde{R}}\left(\frac{15 \tilde{m} v_{0}^{2}}{64 G \sqrt{\tilde{R}}}\right)^{3 / 5},
$$

mit der im Anhang erläuterten Beta-Funktion B, und für die Stoßzahl

$$
\epsilon_{z} \approx 1+\frac{\Delta U_{\mathrm{kin}}}{\tilde{m} v_{0}^{2}} \approx 1-\frac{32}{5} \mathrm{~B}\left(\frac{3}{5}, \frac{3}{2}\right)\left(\frac{15}{64}\right)^{3 / 5} \delta .
$$

Diese Näherungslösung wurde bereits von Kuwabara und Kono [18] angegeben - in der Näherung ist ihr Modell natürlich äquivalent zu der kontaktmechanisch rigorosen Beschreibung. Die weiteren Glieder der Reihenentwicklung von $\epsilon_{z}$ in Potenzen von $\delta$ wurden für das Kuwabara-Kono-Modell von Ramírez et al. [20] und Schwager und Pöschel [21] bestimmt.

Abb.5.4 Stoßzahl als Funktion des bestimmenden Parameters für den Normalstoß einer starren Kugel auf ein Kelvin-Voigt-Medium. MDR-Lösung des rigorosen Modells [24] und Lösung für das Modell von Kuwabara \& Kono

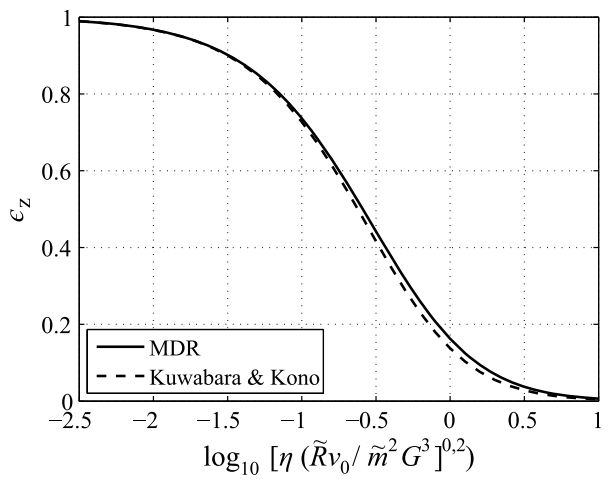


Abb.5.5 Die Größen des in Abb. 5.4 gezeigten Diagramms für sehr kleine Dämpfungsverluste. Die durchgezogene Linie beschreibt die analytische Näherung (5.71)

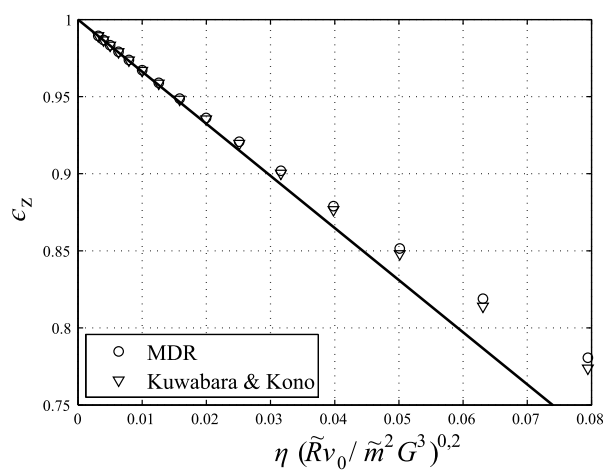

In Abb. 5.5 ist die Stoßzahl nach beiden Modellen für den Bereich sehr kleiner Verluste, gemeinsam mit der Näherung (5.71), gezeigt. Man erkennt, dass die lineare Näherung für $\delta<0,01$ sehr gut mit der vollständigen Lösung übereinstimmt.

\section{Weitere Modelle für den parabolischen Kontakt}

Für den Normalstoß zwischen viskoelastischen Kugeln wurden in der Literatur außerdem mehrere weitere Modelle vorgeschlagen, die zwar aus kontaktmechanischer Sicht zumindest fragwürdig sind, aber durch das Anpassen der freien Parameter auch gut mit experimentellen Ergebnissen in Einklang gebracht werden können und teilweise eigene Vorteile besitzen. Für diese Modelle, die meist aus dem Kontext der Simulation granularer Medien stammen, lässt sich das Kraftgesetz in der Regel verallgemeinert als

$$
F_{z}=-\frac{16 G}{3} \sqrt{\tilde{R} d^{3}}-\lambda d^{\beta} \dot{d}
$$

schreiben. Abgesehen davon, dass bereits der elastische Anteil dieser Kraft während der Restitutionsphase, wie oben beschrieben, nicht ganz korrekt ist, unterscheiden sich die Modelle vor allem in der Wahl des Exponenten $\beta$ des Dämpfungsterms.

Der Fall $\beta=0$ entspricht linearer Dämpfung - der einfachsten Variante, um dem System durch Viskosität Energie zu entziehen - und wurde deswegen von Lee und Herrmann [25] vorgeschlagen, um ein einfaches Stoßmodell für die Integration in molekulardynamische Simulationen granularer Medien zu erhalten. Ray et al. [26] gaben eine analytische Näherungslösung der entstehenden Bewegungsgleichung in Form einer Reihenentwicklung an.

Von Hunt und Crossley [27] stammt der Vorschlag $\beta=3 / 2$, in Korrespondenz zu dem Exponenten in dem Kraftgesetz des elastischen Anteils.

Tsuji et al. [28] untersuchten die Wahl $\beta=1 / 4$. Das hat den Hintergrund, dass der dominierende Parameter für den Stoß eines Flachstempels (siehe oben) durch $\eta / \sqrt{\tilde{m} G a}$ gegeben ist. Wählt man daher 


$$
\lambda d^{\beta}=\alpha(\tilde{m} G \sqrt{\tilde{R} d})^{1 / 2},
$$

wird die sich ergebende Stoßzahl nur von der Wahl des dimensionsfreien Parameters $\alpha$ abhängen und nicht von den Eigenschaften des Systems oder der Stoßgeschwindigkeit.

\subsubsection{Vergleich mit experimentellen Ergebnissen}

Van Zeebroeck et al. [29] untersuchten das Stoßverhalten von Gummi und verschiedenen Biomaterialien in Rückprallversuchen mit einem Pendel. Sie bestimmten dabei die Eindrucktiefe, Geschwindigkeit und Normalkraft als Funktionen der Zeit während der Kollisionen und verglichen ihre experimentellen Ergebnisse mit den Vorhersagen des Modells von Kuwabara und Kono, dessen Parameter die Autor*innen allerdings durch Optimierungsverfahren ermittelten, um eine möglichst gute Übereinstimmung mit den Experimenten zu erzielen. An dieser Stelle sollen die experimentellen Ergebnisse von Van Zeebroeck et al. mit den Vorhersagen des Kuwabara-Kono-Modells und der aus kontaktmechanischer Sicht „korrekteren“ Beschreibung des viskoelastischen Stoßproblems (gelöst durch die MDR) verglichen werden, ohne Gebrauch von optimierten Modellparametern zu machen. Zu diesem Zweck kann man zunächst bemerken, dass die Zeitverläufe der gesuchten Größen unter Verwendung der Skalierung

$$
\hat{d}:=\frac{d}{d_{0}}, \quad d_{0}:=\left(\frac{15 \tilde{m} v_{0}^{2}}{64 G \sqrt{\tilde{R}}}\right)^{2 / 5}, \quad \hat{t}:=\frac{v_{0} t}{d_{0}}, \quad \hat{F}_{z}:=\frac{F_{z} d_{0}}{\tilde{m} v_{0}^{2}}
$$

für beide Modelle nur von dem in Gl. (5.68) definierten Parameter $\delta$, beziehungsweise von der Stoßzahl $\epsilon_{z}$, abhängen. Gibt man daher die Stoßzahl für die Modelle vor, sind die skalierten Verläufe der gesuchten Größen vollständig festgelegt. Zur Reskalierung auf die experimentellen Ergebnisse wurde außerdem angenommen, dass die Anfangsgeschwindigkeit, die maximale Eindrucktiefe und die maximale Normalkraft im Modell mit den experimentell ermittelten Werten übereinstimmen sollen. Ansonsten wurde keine weitere Parameteranpassung vorgenommen.

Van Zeebroeck et al. gaben die Zeitverläufe für die Versuche mit Gummihalbkugeln und (ungeschälten) halbierten Kartoffeln an. Die jeweiligen zur Skalierung der Modelle verwendeten Parameter sind in Tab. 5.1 gegeben.

Tab. 5.1 Die zur Skalierung der theoretischen Modelle verwendeten Größen für den Vergleich mit den experimentellen Ergebnissen von Van Zeebroeck et al.

\begin{tabular}{l|l|l|l|l}
\hline Material & $\epsilon_{z}$ & $v_{0}[\mathrm{~m} / \mathrm{s}]$ & $d_{0}[\mathrm{~mm}]$ & $\tilde{m}[\mathrm{~g}]$ \\
\hline Gummi & 0,71 & 0,2 & 1,06 & 279 \\
\hline Kartoffel & 0,56 & 0,4 & 1,51 & 320 \\
\hline
\end{tabular}


Die Abb.5.6 und 5.7 zeigen die Verläufe der Eindrucktiefe als Funktion der Zeit für die beiden theoretischen Modelle im Vergleich mit den experimentellen Ergebnissen. Die Übereinstimmung zwischen der theoretischen Vorhersage und dem Experiment ist sehr gut, dabei unterscheiden sich die beiden untersuchten Modelle nur sehr geringfügig voneinander. Diese überschätzen die Stoßdauer, außerdem ist die Übereinstimmung bei den Versuchen mit Gummi besser als bei denen mit den halbierten Kartoffeln. Dies ist vermutlich auf deren unregelmäßigere Form und den Einfluss der Schale zurückzuführen.

Die Übereinstimmung zwischen Theorie und Experiment für die Verläufe der Eindruckgeschwindigkeit ist ebenso gut wie im Fall der Eindrucktiefe, da in den Experimenten die Geschwindigkeit nicht separat gemessen, sondern durch diskrete Differenzierung aus den Verschiebungsdaten bestimmt wurde. Die entsprechenden Diagramme sind deswegen an dieser Stelle aus Platzgründen ausgespart.

In den Abb. 5.8 und 5.9 sind die Verläufe der im Experiment gesondert gemessenen Normalkräfte und die entsprechenden theoretischen Vorhersagen gezeigt. Die Übereinstimmung ist zwar schlechter als bei den Verschiebungen und Geschwindigkeiten (besonders im Fall der Experimente mit Kartoffelhälften), aber trotzdem noch zufriedenstellend. Insbesondere muss man kritisch anmerken, dass im Experiment der Zeitpunkt der Bewegungsumkehr am

Abb.5.6 Verlauf der Eindrucktiefe als Funktion der Zeit für den Stoß einer Gummihalbkugel auf eine starre Platte. Theoretische Vorhersage und experimentelle Ergebnisse

Abb.5.7 Verlauf der Eindrucktiefe als Funktion der Zeit für den Stoß einer Kartoffelhälfte auf eine starre Platte. Theoretische Vorhersage und experimentelle Ergebnisse
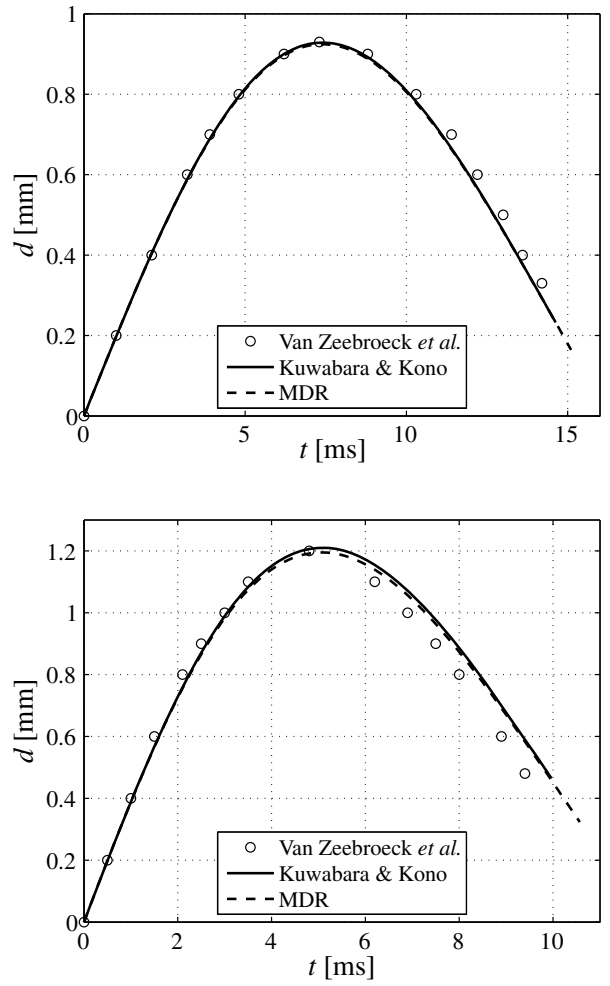
Abb.5.8 Verlauf der Normalkraft als Funktion der Zeit für den Stoß einer Gummihalbkugel auf eine starre Platte. Theoretische Vorhersage und experimentelle Ergebnisse

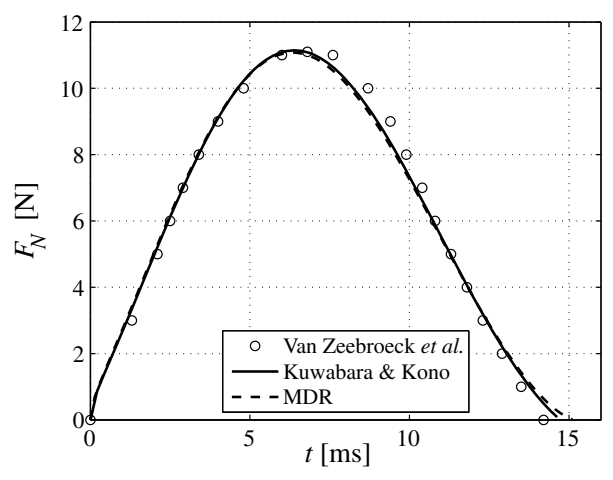

Abb. 5.9 Verlauf der Normalkraft als Funktion der Zeit für den Stoß einer

Kartoffelhälfte auf eine starre Platte. Theoretische Vorhersage und experimentelle Ergebnisse

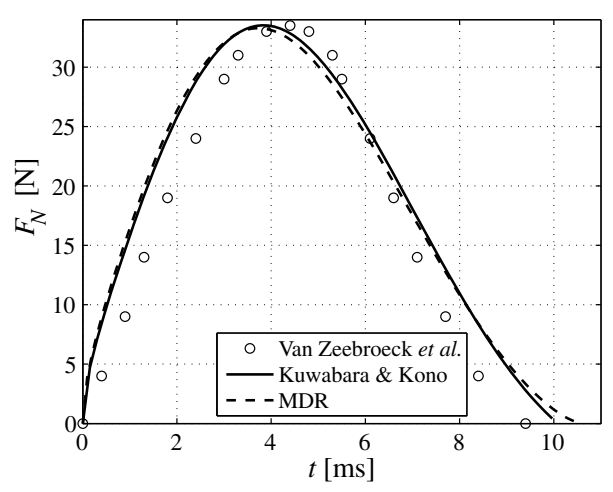

Ende der Kompressionsphase nicht mit dem Zeitpunkt der maximalen Normalkraft übereinstimmt; dadurch fällt die Normalkraft zu Beginn der Restitutionsphase in den Modellen systematisch kleiner aus als im Experiment gemessen.

\subsubsection{Inkompressibles Standardmedium}

Das Kelvin-Voigt-Medium mit seiner unendlich schnellen Spannungsrelaxation und der damit einhergehenden Separierung von elastischen und viskosen Eigenschaften ist nur eine sehr grobe Näherung tatsächlichen viskoelastischen Materialverhaltens. Die nächstbessere Näherung besteht in der Berücksichtigung der finiten Zeitskala der Spannungsrelaxation; es ergibt sich dann das sogenannte Standardmedium mit dem in Gl.(3.191) gegebenen zeitabhängigen Schubmodul

$$
G(t)=G_{\infty}+G_{1} \exp \left(-\frac{t}{\tau}\right), \quad \tau:=\frac{\eta}{G_{1}} .
$$


Im Folgenden wird für dieses Materialmodell wiederum das Normalstoßproblem mit einem starren Rückprallkörper der Masse $\tilde{m}$ und der Anfangsgeschwindigkeit $v_{0}$ betrachtet, wobei als Rückprallkörper ein zylindrischer Flachstempel und eine Kugel in der parabolischen Profilnäherung Verwendung finden.

\section{Flachstempel-Kontakt}

Da der Kontakt mit einem starren zylindrischen Stempel mit dem Radius $a$ vollständig linear ist, kann er durch ein einzelnes lineares rheologisches Element abgebildet werden. Das in Abb. 5.10 gezeigte Modell ist daher ein exaktes Abbild des zu untersuchenden Stoßproblems.

Die Bewegungsgleichungen für die beiden Freiheitsgrade $d$ und $\tilde{d}$ sind

$$
\begin{aligned}
& 0=\tilde{m} \ddot{d}+8 G_{\infty} a d+8 G_{1} a d, \\
& 0=G_{1} \tilde{d}+\eta(\dot{\tilde{d}}-\dot{d}) .
\end{aligned}
$$

Mit den dimensionslosen Größen

$$
\hat{t}:=\sqrt{\frac{8 a G_{\infty}}{\tilde{m}}} t, \quad \hat{d}:=\frac{1}{v_{0}} \sqrt{\frac{8 a G_{\infty}}{\tilde{m}}} d
$$

kann dies in die Gleichung dritter Ordnung

$$
0=\frac{\mathrm{d}^{3} \hat{d}}{\mathrm{~d} \hat{t}^{3}}+\frac{1}{\beta \delta} \frac{\mathrm{d}^{2} \hat{d}}{\mathrm{~d} \hat{t}^{2}}+\left(1+\frac{1}{\beta}\right) \frac{\mathrm{d} \hat{d}}{\mathrm{~d} \hat{t}}+\frac{1}{\beta \delta} \hat{d}, \quad \beta:=\frac{G_{\infty}}{G_{1}}, \quad \delta:=\eta \sqrt{\frac{8 a}{\tilde{m} G_{\infty}}},
$$

mit den Anfangsbedingungen

$$
\hat{d}(0)=0, \quad \frac{\mathrm{d} \hat{d}}{\mathrm{~d} \hat{t}}(0)=1, \quad \frac{\mathrm{d}^{2} \hat{d}}{\mathrm{~d} \hat{t}^{2}}(0)=0,
$$

umgeformt werden. Der Stoß endet, falls die Normalkraft verschwindet, das heißt, wenn

$$
\frac{\mathrm{d}^{2} \hat{d}}{\mathrm{~d} \hat{t}^{2}}\left(\hat{T}_{S}\right)=0, \quad T_{S}>0 .
$$

Die zu Gl. (5.79) gehörige voll-kubische Eigenwertgleichung kann man theoretisch geschlossen analytisch lösen, wie Argatov [17] demonstrierte, allerdings ist das Ergebnis und die

Abb. 5.10 Rheologisches Modell des Normalstoßes eines starren zylindrischen Stempels auf ein Standardmedium

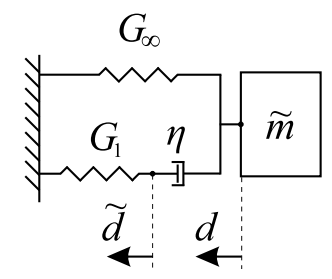


Abb.5.11 KonturlinienDiagramm der Stoßzahl eines starren zylindrischen Stempels auf ein Standardmedium in Abhängigkeit der beiden definierenden dimensionslosen Parameter in logarithmischer Skalierung

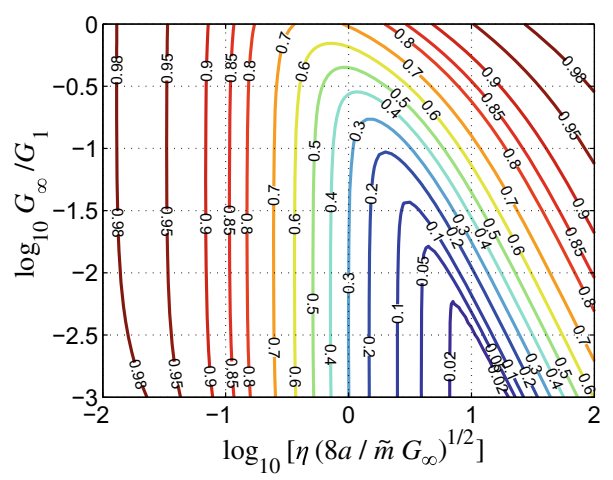

Bestimmung der sich ergebenden Stoßzahl so umständlich, dass eine numerische Lösung einfacher erscheint. Die Stoßzahl als Funktion der beiden einzigen dimensionslosen Parameter $\beta$ und $\delta$ ist als doppel-logarithmisches Konturlinien-Diagramm in Abb. 5.11 gezeigt. Für $\beta=0$ ergibt sich das oben bereits untersuchte Kelvin-Voigt-Medium. Da der statische Modul von Elastomeren in der Regel mehrere Größenordnungen kleiner ist als der Glasmodul, wurden nur Werte von $\beta$ zwischen $10^{-3}$ und Eins berücksichtigt. Man erkennt, dass für Werte $\delta<1$ die Stoßzahl fast nicht von $\beta$ abhängt, das Verhalten also mit dem des Kelvin-Voigt-Mediums zusammenfällt. Für sehr große Werte von $\delta$ nimmt die Stoßzahl wieder zu. Dies liegt daran, dass sich das Standardmedium auf sehr kurzen Zeitskalen wie ein elastischer Körper verhält. Warum man dies in Experimenten nicht beobachtet, wird bei der Betrachtung des Kelvin-Maxwell-Mediums im nächsten Abschnitt deutlich.

Von Butcher und Segalman [15] und Argatov [17] stammen asymptotische Störungsrechnungen erster Ordnung für das Stoßproblem in der Nähe des Kelvin-Voigt-Mediums $\left(G_{1} \rightarrow \infty\right)$ und von Argatov [17] in der Nähe des Maxwell-Mediums $\left(G_{\infty} \rightarrow 0\right)$.

\section{Lösung durch Rückführung auf das Kelvin-Voigt-Medium}

Da der komplexe Modul eines viskoelastischen Mediums, abgesehen von Materialparametern, nur von der Frequenz abhängt, kann jedes viskoelastische Medium bei einer harmonischen Anregung mit konstanter Kreisfrequenz $\omega_{0}$ als Kelvin-Voigt-Medium betrachtet werden, indem der Realteil des komplexen Moduls bei der gegebenen Frequenz (der Speichermodul) als $G_{\infty}$ und der Imaginärteil (der Verlustmodul) als $\eta \omega_{0}$ eines Kelvin-VoigtMediums gedeutet werden.

Die Indentierungstiefe in viskoelastischen Stößen folgt im Allgemeinen keinem exakt harmonischen Zeitverlauf; allerdings haben Stoßprobleme eine charakteristische Zeitskala, daher liegt es nahe, das allgemeine viskoelastische Stoßproblem durch die Rückführung auf ein Problem mit einem geeigneten Kelvin-Voigt-Medium zu lösen. Dies soll hier als Beispiel anhand des Stoßes eines Flachstempels auf ein Standard-Medium demonstriert werden, für den man oben die exakte Lösung nachschlagen kann. 
Wählt man als charakteristische Zeitskala die Dauer des elastischen Stoßes mit $G=G_{\infty}$ ist die charakteristische Kreisfrequenz der Bewegung durch

$$
\omega_{0}=\sqrt{\frac{8 G_{\infty} a}{\tilde{m}}}
$$

bestimmt. Mit dem in Gl.(3.193) gegebenen komplexen Modul des Standardmediums und den in Gl.(5.79) eingeführten dimensionslosen Parametern $\beta$ und $\delta$ ist das in Gl.(5.56) eingeführte Dämpfungsmaß

$$
D=\frac{\delta}{2} \frac{1}{1+\delta^{2} \beta^{2}+\delta^{2} \beta},
$$

welches dann in die in den Gl.(5.61) und (5.62) gegebene Lösung für das Kelvin-VoigtMedium eingesetzt werden kann. Für die Stoßzahl als Funktion von $\beta$ und $\delta$ erhält man beispielsweise die in Abb.5.12 dargestellte Lösung.

Man erkennt, dass die Lösung in sehr großen Parameterbereichen sehr gut mit der exakten Lösung übereinstimmt. Nur für sehr große Werte von $\delta$ und gleichzeitig kleine Werte von $\beta$ gibt es stärkere Abweichungen. Das liegt daran, dass bei diesen Parameterkombinationen die tatsächliche Stoßdauer zu stark von dem elastischen Fall mit $G=G_{\infty}$ abweicht.

Die geschilderte Methode hat den großen Vorteil, vollständig analytisch und dabei sehr leicht handhabbar zu sein und wegen der sehr allgemeinen Form von Gl.(5.56) leicht für beliebige viskoelastische Rheologien verallgemeinert werden zu können.

\section{Parabolischer Kontakt}

Das Gleichungssystem, um den Stoß eines parabolischen Rückprallkörpers mit dem Radius $\tilde{R}$ auf ein Standardmedium kontaktmechanisch rigoros zu untersuchen, wird gebildet durch die allgemeine Bewegungsgleichung (2.34), die elastische Kontaktlösung aus den Gl. (3.35) und (3.36), die Materialfunktion (3.191) und die allgemeinen viskoelastischen Beziehungen (3.202) und (3.203) für die Kompressionsphase sowie (3.206) und (3.208) für die

Abb. 5.12 KonturlinienDiagramm der Stoßzahl eines starren zylindrischen Stempels auf ein Standardmedium in Abhängigkeit der beiden definierenden dimensionslosen Parameter in logarithmischer Skalierung. Näherungslösung mithilfe der Rückführung auf ein Kelvin-Voigt-Medium

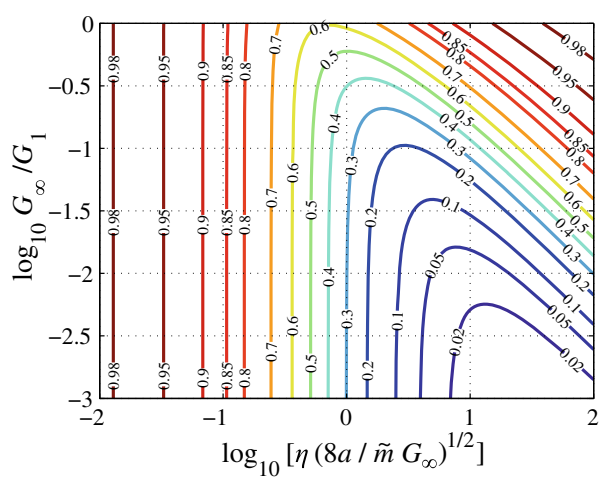


Restitutionsphase. Zur Lösung dieses gewöhnlichen aber komplizierten Integro-DifferentialGleichungssystems bietet sich ein MDR-Modell an, das von Willert et al. [24] und Kusche [30] beschrieben wurde, und das diese Gleichungen exakt reproduziert.

Es wird der ebene Körper mit dem Profil $g(x)=x^{2} / \tilde{R}$ in die Bettung aus unabhängigen, linearen rheologischen Elementen, die das Relaxationsverhalten des Standardmediums reproduzieren, eingedrückt. Die vertikalen Verschiebungen der Elemente im Kontakt sind durch

$$
u_{z}(x, t)=-d(t)+g(x), \quad|x| \leq a(t)
$$

gegeben. Während der Kompressionsphase kommt ein Element dabei geometrisch in Kontakt, d.h. wenn es durch den Eindruckkörper ausgelenkt wird. Zur Bestimmung der Streckenlast $q_{z}$ aus den Verschiebungen sind zwei Wege möglich. Einerseits ist

$$
q_{z}(x, t)=4 G_{\infty} u_{z}(x, t)+4 G_{1} \int_{0}^{t} \exp \left(\frac{t^{\prime}-t}{\tau}\right) \dot{u}_{z}\left(x, t^{\prime}\right) \mathrm{d} t^{\prime}, \quad \tau:=\frac{\eta}{G_{1}} .
$$

Wird der Integral-Anteil aus der Relaxation in äquidistanten diskreten Zeitschritten $t_{j}=j \Delta t$ ausgewertet, ergibt sich die Summe

$$
q_{z, j}^{\text {relax }}(x)=4 G_{1} \Delta t \sum_{i=0}^{j} \exp \left(\frac{t_{i}-t_{j}}{\tau}\right) \dot{u}_{z, i}(x)+\mathcal{O}\left(\Delta t^{2}\right) .
$$

Durch eine einfache Indexverschiebung erhält man dann die Rekursionsbeziehung

$$
q_{z, j+1}^{\text {relax }}(x)=\exp \left(-\frac{\Delta t}{\tau}\right) q_{z, j}^{\text {relax }}(x)+4 G_{1} \dot{u}_{z, j+1}(x) \Delta t+\mathcal{O}\left(\Delta t^{2}\right) .
$$

Eine zweite Variante zur Bestimmung der Streckenlast besteht in der Auswertung der Gleichgewichtsbeziehungen der äußeren und inneren Freiheitsgrade jedes Elements der Bettung ${ }^{9}$,

$$
\begin{gathered}
0=\tilde{u}_{z}+\tau\left(\dot{\tilde{u}}_{z}-\dot{u}_{z}\right) \\
q_{z}=4 G_{\infty} u_{z}+4 G_{1} \tilde{u}_{z} .
\end{gathered}
$$

Während der Restitutionsphase verlässt ein Element den Kontakt, wenn $q_{z}>0$. Die gesamte Normalkraft ergibt sich, wie immer in der MDR, als Integral der Streckenlast.

Durch Dimensionsanalysen und numerische Experimente kann man zeigen, dass die Stoßzahl nur von den beiden Parametern

\footnotetext{
${ }^{9}$ Zur Erläuterung kann wiederum Abb. 5.10 herangezogen werden, wobei auf das rheologische Element nicht die ganze Normalkraft wirkt, sondern nur der infinitesimale Anteil $q_{z} \mathrm{~d} x$.
} 
Abb.5.13 KonturlinienDiagramm der Stoßzahl eines starren Paraboloids auf ein Standardmedium in Abhängigkeit der beiden definierenden dimensionslosen Parameter in logarithmischer Skalierung. Alle freien Input-Größen wurden zufällig generiert

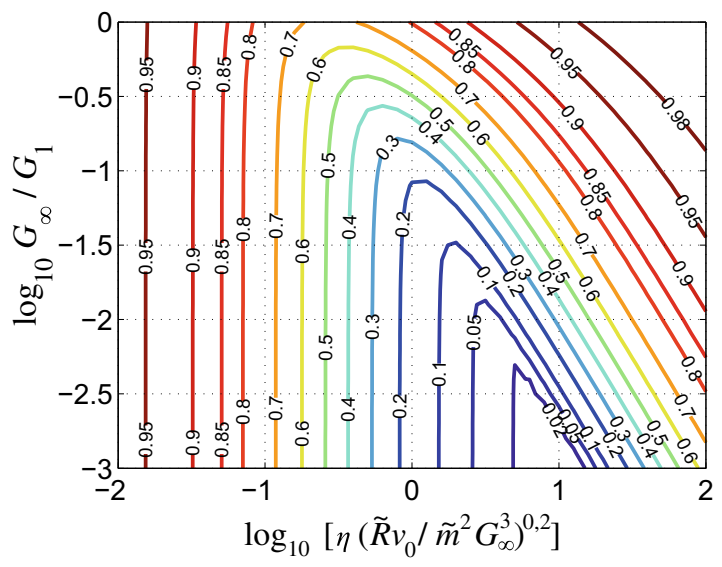

$\beta:=\frac{G_{\infty}}{G_{1}}, \quad \delta:=\eta\left(\frac{\tilde{R} v_{0}}{\tilde{m}^{2} G_{\infty}^{3}}\right)^{1 / 5}$

abhängt. Diese haben die gleiche Bedeutung, wie die beiden Parameter, die oben für den Stoß eines Flachstempels verwendet wurden. Die Lösung für die Stoßzahl als Funktion der bestimmenden Größen ist in Abb. 5.13 gezeigt. Interessanterweise unterscheidet sich die Lösung wiederum nur sehr geringfügig von dem in Abb.5.11 gezeigten Fall des Flachstempels. Wie für den elastischen Normalstoß hat also auch für das viskoelastische Stoßproblem die konkrete Form des Rückprallkörpers nur eine untergeordnete Bedeutung, die Stoßzahl wird hauptsächlich durch die Materialeigenschaften des viskoelastischen Mediums bestimmt. In den folgenden Abschnitten kommt daher nur noch der parabolische Fall zur Anwendung.

\subsubsection{Inkompressibles Kelvin-Maxwell-Medium}

Das Kelvin-Maxwell-Medium kann man als (mehr oder weniger gleichwertigen) Ersatz einer vollständigen Prony-Reihe verwenden, wenn das viskoelastische Kontaktproblem selbst eine feste inhärente Zeitskala hat (für Stoßprozesse ist das natürlich die Stoßdauer $T_{S}$ ). Die Berücksichtigung der Relaxation in ihrem tatsächlichen Zeitverlauf ist dann nur für die Relaxationsprozesse nötig, deren charakteristische Dauer mit der Zeitskala des Kontaktproblems zusammenfällt. Deutlich schnellere Relaxationen können in guter Näherung als unendlich schnell, deutlich langsamere entsprechend als unendlich langsam angenommen werden. Von der gesamten Prony-Reihe (in Form eines generalisierten Maxwell-Mediums) bleibt dann nur ein einziges Maxwell-Element mit dem Modul $G_{1}$ übrig, dessen Relaxationszeit durch die Zeitskala des Kontaktproblems gegeben ist. Die Maxwell-Elemente mit schnellerer Relaxation fast man zu einem einzelnen Dämpfer mit der Viskosität $\eta_{0}$ zusammen, die Moduln $G_{i}$ der langsamen Relaxationsprozesse schlägt man dem statischen Modul $G_{\infty} \mathrm{zu}-$ 
Abb.5.14 KonturlinienDiagramm der Stoßzahl eines starren Paraboloids auf ein Kelvin-Maxwell-Medium in Abhängigkeit der beiden definierenden dimensionslosen Parameter in logarithmischer Skalierung. Alle freien Input-Größen wurden zufällig generiert

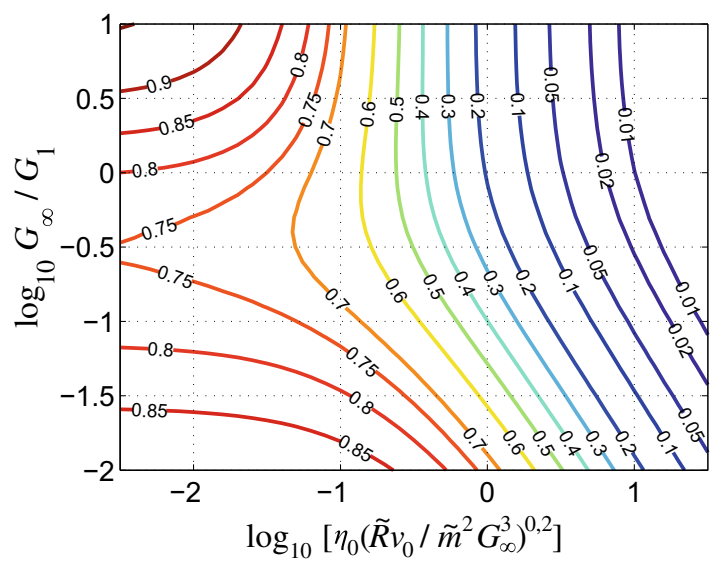

siehe die Gl. (3.196) und (3.197). Der zeitabhängige Modul des so definierten rheologischen Modells ist durch Gl.(3.198) gegeben. Für die Relaxationszeit $\tau$ des Maxwell-Elements wird die elastische Stoßdauer nach Gl.(5.12) mit $G=G_{\infty}$ vorgegeben ${ }^{10}$.

An der numerischen Lösung des Problems im Rahmen der MDR ändert sich gegenüber dem im vorhergehenden Abschnitt, zur Behandlung des Standardmediums, beschriebenen Algorithmus nicht viel; man muss nur die Gleichgewichtsbeziehung (5.89) durch den Zusammenhang

$$
q_{z}=4 G_{\infty} u_{z}+4 G_{1} \tilde{u}_{z}+4 \eta_{0} \dot{u}_{z}
$$

ersetzen. Durch numerische Studien kann man leicht beweisen, dass die Stoßzahl nur von den beiden dimensionslosen Parametern

$$
\beta:=\frac{G_{\infty}}{G_{1}}, \quad \delta:=\eta_{0}\left(\frac{\tilde{R} v_{0}}{\tilde{m}^{2} G_{\infty}^{3}}\right)^{1 / 5}
$$

abhängt. Diese Abhängigkeit ist in Abb. 5.14 in logarithmischer Darstellung gezeigt.

Da die Moduln mehrerer Relaxationszeiten zu $G_{\infty}$ zusammengefasst werden und $G_{1}$ nur dem Modul einer einzelnen Relaxationszeit (der elastischen Stoßdauer) entspricht, wurde ein etwas anderer Parameterbereich für $\beta$ gewählt als in Abb. 5.13 für das Standardmedium. Man erkennt, dass die Stoßzahl im Bereich sehr großer Werte von $\delta$ (also beispielsweise für sehr große Geschwindigkeiten) nicht, wie im Fall des Standardmediums, zur elastischen

\footnotetext{
${ }^{10}$ Bei der Verwendung der tatsächlichen viskoelastischen Stoßdauer würde sich die Problemstellung transzendieren; die Lösung des sich ergebenden Fixpunkt-Problems wäre nicht sonderlich hilfreich, besonders da in technischen Prony-Reihen die Relaxationszeiten kein kontinuierliches Spektrum bilden, sondern nach Größenordnungen sortiert sind.
} 
Lösung zurückkehrt, sondern, durch die Wirkung des einzelnen Dämpfers, d.h. der Wirkung der schnelleren Relaxationsprozesse, gegen Null konvergiert, wie im Fall des Kelvin-VoigtMediums.

\subsubsection{Kompressibles Kelvin-Voigt-Medium}

Die Berücksichtigung der Kompressibilität in viskoelastischen Kontaktproblemen ist in der Regel kompliziert. Mithilfe der im dritten Kapitel gezeigten exakten Rückführung reibungsfreier Normalkontakte kompressibler viskoelastischer Medien auf ein äquivalentes inkompressibles Problem ist allerdings die Untersuchung des reinen Normalstoßproblems grundsätzlich ohne größere Schwierigkeiten möglich.

Brilliantov et al. [31] führten physikalisch rigorose, störungstheoretische Rechnungen bis zur ersten Ordnung durch, um den dissipativen Anteil der Normalkraft während des Normalstoßes einer starren Kugel auf ein allgemeines (kompressibles) Kelvin-Voigt-Medium zu untersuchen, ohne allerdings das Stoßproblem selbst genauer zu betrachten. Willert et al. [32] untersuchten den Normalstoß einer starren Kugel auf ein kompressibles Standardmedium bei rein elastischer Reaktion gegen hydrostatischen Druck. Da die konkrete Rheologie und das Profil des Eindruckkörpers für das quasistatische, viskoelastische Normalstoßproblem, wie oben beschrieben, nur eine untergeordnete Rolle spielen, soll an dieser Stelle der einfachste allgemeine Fall einer Kollision zwischen einem starren zylindrischen Flachstempel mit einem allgemeinen Kelvin-Voigt-Körper, der durch die zeitabhängigen Schub- und Kompressionsmoduln,

$$
\begin{aligned}
& G(t)=G_{\infty}+\eta \delta(t), \\
& K(t)=K_{\infty}+\xi \delta(t),
\end{aligned}
$$

charakterisiert ist, beschrieben werden. In diesen Gleichungen bezeichnen $G_{\infty}$ und $K_{\infty}$ die jeweiligen statischen Moduln, $\eta$ und $\xi$ die Scher-, beziehungsweise Volumenviskosität und $\delta(t)$ die Dirac-Distribution. Der Flachstempel habe den Radius $a$, die Masse $\tilde{m}$ und die Anfangsgeschwindigkeit $v_{0}$.

Als Maß der Kompressibilität diene die statische Poissonzahl ${ }^{11}$

$$
v:=\frac{3 K_{\infty}-2 G_{\infty}}{6 K_{\infty}+2 G_{\infty}}
$$

Mithilfe des in Abb.3.11 gezeigten rheologischen Modells eines allgemeinen kompressiblen viskoelastischen Mediums erhält man sofort das in Abb. 5.15 dargestellte rheologische Modell des Stoßproblems.

\footnotetext{
${ }^{11}$ Es sei in diesem Zusammenhang darauf hingewiesen, dass die Angabe einer dynamischen (zeitabhängigen) Poissonzahl für ein viskoelastisches Material im Allgemeinen nicht ganz elementar ist [33].
} 
Abb. 5.15 Rheologisches Modell des Normalstoßes eines starren zylindrischen Stempels auf ein allgemeines kompressibles Kelvin-Voigt-Medium

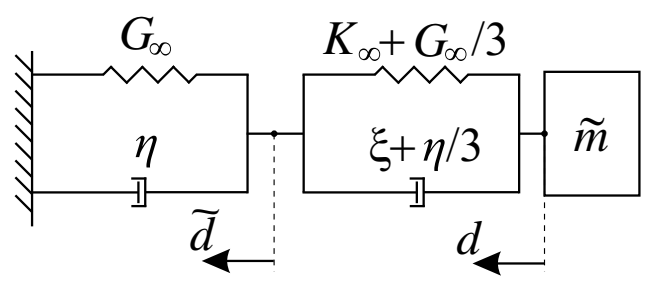

Die Bewegungsgleichungen für die beiden Freiheitsgrade $d$ und $\tilde{d}$ sind durch

$$
\begin{gathered}
0=\tilde{m} \ddot{d}+8 \eta a \dot{\tilde{d}}+8 G_{\infty} a \tilde{u}, \\
0=(3 \xi+4 \eta) \dot{\tilde{d}}+(3 K+4 G) \tilde{d}-(3 \xi+\eta) \dot{d}-(3 K+G) d
\end{gathered}
$$

gegeben. Durch Einführung der dimensionsfreien Größen

$$
x:=\frac{d \omega_{0}}{v_{0}}, \quad \tilde{x}:=\frac{\tilde{d} \omega_{0}}{v_{0}}, \quad \tilde{t}:=\omega_{0} t, \quad \omega_{0}:=\sqrt{\frac{8 G_{\infty} a}{\tilde{m}}}
$$

kann man leicht zeigen, dass das Verhalten des Systems in skalierten Größen nur von den drei dimensionsfreien Parametern

$$
v, \quad 2 D_{1}:=\frac{\eta}{G_{\infty}} \omega_{0}, \quad 2 D_{2}:=\frac{3 \xi+\eta}{3 K_{\infty}+G_{\infty}} \omega_{0}
$$

abhängt. Die Lösung des Bewegungsgleichungssystems bereitet dabei keine Probleme. Um die Anzahl an freien Parametern für die Darstellung der Lösung möglichst gering zu halten, sei angenommen, dass die Scher- und Volumenviskosität des viskoelastischen Materials gleich sind ${ }^{12}$. In diesem Fall ist

$$
D_{2}=\frac{4}{3}(1-2 v) D_{1}
$$

Die Stoßzahl als Funktion der beiden verbleibenden Parameter ist als Kurvenschar in Abb.5.16 dargestellt. Offenbar hat auch die Kompressibilität nur einen vergleichsweise geringen Einfluss auf das Dissipationsverhalten während der Kollision. Am stärksten ist der Einfluss im Bereich mittlerer Dämpfungen, wo die relative Differenz zwischen der Lösung für $v=0,3$ und dem inkompressiblen Grenzfall etwa $10 \%$ beträgt. Dabei ist die Stoßzahl im inkompressiblen Grenzfall grundsätzlich am kleinsten.

\footnotetext{
${ }^{12}$ Diese Annahme ist durchaus gerechtfertigt, da sich in vielen Polymeren Scher- und Volumenviskosität deutlich weniger voneinander unterscheiden als der Schub- und Kompressionsmodul [34].
} 


\author{
Abb.5.16 Stoßzahl für den \\ Normalstoß eines zylindrischen \\ Flachstempels auf ein \\ kompressibles \\ Kelvin-Voigt-Medium als \\ Funktion der dimensionsfreien \\ Dämpfung $D_{1}$ für verschiedene \\ statische Poissonzahlen bei \\ gleicher Volumen- und \\ Scherviskosität. Die dünne \\ durchgezogene Linie \\ bezeichnet den \\ inkompressiblen Grenzfall \\ (siehe auch Abb. 5.3)
}

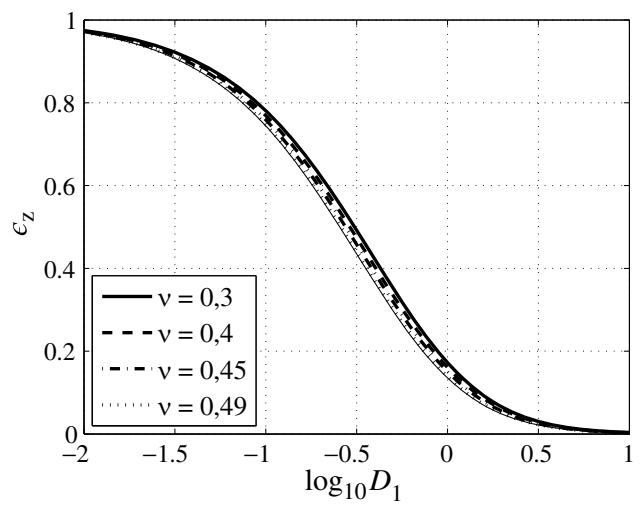

\subsection{Elasto-Plastischer Normalstoß ohne Adhäsion}

Da für viele metallische Werkstoffe schon Stoßgeschwindigkeiten der Größenordnung $0,01 \mathrm{~m} / \mathrm{s}$ ausreichen, um plastische Deformationen zu initiieren [35, S. 130], laufen die Kollisionen in den allermeisten Fällen nicht ideal-elastisch ab, sondern snd mit einem gewissen plastischen Energieverlust verbunden. Zum (elasto-)plastischen Normalstoß zwischen Kugeln oder zwischen einer Kugel mit einem Halbraum gibt es dabei inzwischen eine recht umfangreiche Literatur.

Weir und Tallon [36] untersuchten den elastisch-ideal-plastischen Zusammenstoß von Kugeln und stellten unter anderem theoretisch und experimentell fest, dass bei wiederholten Kollisionen die Stoßzahl wegen der permanenten Deformation aus den vorherigen Zusammenstößen steigt. In einer Serie von Publikationen [37-39] studierten Wu et al. das Problem mithilfe der Finite-Elemente-Methode (FEM) und bestimmten unter anderem die Stoßgeschwindigkeit, die nötig ist, um den Bereich unbeschränkten plastischen Fließens zu erreichen. Da bei solch hohen Geschwindigkeiten dynamische Effekte relevant werden, zogen sie auch die Dissipation durch die Abstrahlung elastischer Wellen in Betracht und kamen dabei zu vergleichbaren Ergebnissen wie Hunter [4]. Thornton et al. [40] verglichen das Modell von Thornton für den elasto-plastischen Stoß mit mehreren kontaktmechanisch nicht rigorosen Feder- und Feder-Dämpfer-Modellen.

Wenn die kollidierenden Teilchen sehr klein sind, muss man bei der Untersuchung des Stoßproblems die Skaleneffekte der Kristallplastizität berücksichtigen, das Fließkriterium ist dann nicht-lokal. Für den Fall, dass die gemessene Härte umgekehrt proportional zum Kontaktradius ist ${ }^{13}$, lösten Lyashenko und Popov [42] das entsprechende elasto-plastische Normalstoßproblem.

\footnotetext{
${ }^{13}$ Bei einem parabolischen Indenter bedeutet dies, dass das Quadrat der Härte umgekehrt proportional zur Eindrucktiefe ist; diese Abhängigkeit ist für die Mikroindentierung charakteristisch [41, S. 44]).
} 


\subsubsection{Theoretische Modellierung}

Mithilfe der drei im Abschn.3.8.2 geschilderten theoretischen Modelle für den elastoplastischen Normalkontakt von Kugeln soll im vorliegenden Abschnitt das entsprechende quasistatische Stoßproblem gelöst werden. Die theoretischen Vorhersagen werden anschließend mit experimentellen Ergebnissen verglichen.

\section{Modell von Thornton}

Durch Gl. (3.278) ist der hysteretische Energieverlust für einen vollständigen Belastungszyklus des Normalkontaktes elasto-plastischer Kugeln nach dem Modell von Thornton gegeben. Damit kann auch das entsprechende Stoßproblem ohne Schwierigkeiten gelöst werden [43]. Ohne Beschränkung der Allgemeinheit ${ }^{14}$ betrachte man eine starre Kugel der Masse $\tilde{m}$, die mit der Geschwindigkeit $v_{0}$ auf einen elasto-plastischen Halbraum trifft.

Der erste Term in Gl. (3.278) beschreibt die Arbeit, die während der Kompressionsphase verrichtet wird. Daraus folgt die bestimmende Gleichung zur Ermittlung der maximalen Eindrucktiefe:

$$
\frac{\tilde{m} v_{0}^{2}}{2 F_{Y} d_{Y}}=\frac{2}{5}+\frac{1}{4}\left(\frac{3 d_{\max }^{2}}{d_{Y}^{2}}-2 \frac{d_{\max }}{d_{Y}}-1\right) .
$$

Dabei ist wegen Gl.(3.266)

$$
F_{Y} d_{Y}=\frac{4}{3}\left(\frac{4 \pi}{5}\right)^{5}\left(\frac{\sigma_{f}}{\tilde{E}}\right)^{5} \tilde{E} \tilde{R}^{3} .
$$

Die kritische Geschwindigkeit $v_{Y}$, die nötig ist, um plastische Deformationen zu initiieren, ergibt sich aus der Bedingung $d_{\max }=d_{Y}$, also

$$
v_{Y}^{2}=\frac{16}{15}\left(\frac{4 \pi}{5}\right)^{5}\left(\frac{\sigma_{f}}{\tilde{E}}\right)^{5} \frac{\tilde{E} \tilde{R}^{3}}{\tilde{m}} .
$$

Alle Ergebnisse in diesem Unterkapitel beziehen sich natürlich auf den Fall $v_{0} \geq v_{Y}$. Aus den obigen Gleichungen kann man die explizite Beziehung

$$
\frac{d_{\max }}{d_{Y}}=\frac{1}{3}+\frac{2}{15} \sqrt{\frac{30 v_{0}^{2}}{v_{Y}^{2}}-5}
$$

für die maximale Eindrucktiefe während der Kollision herleiten, aus der sich mithilfe der Kontaktlösung die gesamte Kontaktkonfiguration am Ende der Kompressionsphase ergibt.

Für die Stoßzahl erhält man wegen Gl.(3.278)

\footnotetext{
${ }^{14}$ Im Thorntonschen Modell gibt es keinen Unterschied zwischen dem Kontakt eines starren Eindruckkörpers mit einem elasto-plastischen Halbraum und dem elasto-plastischer Körper.
} 


$$
\epsilon_{z}=\frac{v_{Y}}{v_{0}}\left[\frac{R_{p}}{\tilde{R}}\left(\frac{d_{\mathrm{max}}-d_{\mathrm{res}}}{d_{Y}}\right)^{5}\right]^{1 / 4}
$$

Dabei sind der Krümmungsradius $R_{p}$ während der Restitution und die Resteindrucktiefe $d_{\text {res }}$ durch die Gl. (3.273) und (3.274) gegeben. Für sehr große Geschwindigkeiten $v_{0}$ erhält man die asymptotische Näherung

$$
\epsilon_{z} \approx 1,185\left(\frac{v_{0}}{v_{Y}}\right)^{-1 / 4}
$$

\section{Interpolation im elasto-plastischen Bereich}

Da auch im Interpolationsmodell die Entlastung als ein elastischer Prozess mit einem durch die vorangegangene plastische Deformation veränderten Krümmungsradius betrachtet wird, behält Gl.(5.105) für die Stoßzahl ihre Gültigkeit. Allerdings muss die maximale Eindrucktiefe wegen Gl.(3.288) als Lösung der Gleichung

$$
\frac{v_{0}^{2}}{v_{Y}^{2}}=1+\frac{5}{2}\left(\frac{d_{\max }}{d_{Y}}-1\right)+\frac{15}{8}\left(\frac{d_{\max }}{d_{Y}}-1\right)^{2}+\frac{5 C_{2}}{6}\left(\frac{d_{\max }}{d_{Y}}-1\right)^{3}+\frac{5 C_{3}}{8}\left(\frac{d_{\max }}{d_{Y}}-1\right)^{4}
$$

bestimmt werden, wobei die Konstanten $C_{2}$ und $C_{3}$ in Gl.(3.285) gegeben sind. Es sei außerdem angemerkt, dass die obige Gl.(5.107) nur gültig ist, falls nicht in den voll-plastischen Bereich hinein belastet wird. Die Änderungen, die sich bei der Belastung in den vollplastischen Bereich ergeben, bereiten allerdings keine Schwierigkeiten und wurden nur aus Platzgründen ausgespart. Die Werte von $R_{p}$ und $d_{\text {res }}$ ergeben sich unter Zuhilfenahme der Zusammenhänge (3.284) und (3.287) aus den Gl. (3.273) und (3.274).

\section{Analytische Approximation von FEM-Lösungen}

Die in Abschn. 3.8.2 dargestellte, durch die analytische Approximation von rigorosen FEMLösungen gewonnene Lösung von Jackson et al. [44] für den Normalkontakt einer elastoplastischen Kugel mit einem elasto-plastischen Halbraum kann man ohne Schwierigkeiten in die Bewegungsgleichung der Kugel einsetzen. Durch numerische Integration des entstehenden Gleichungssystems löst man das entsprechende Stoßproblem. Da, wie im dritten Kapitel beschrieben, für den elasto-plastischen Kontakt die makroskopische Form der kontaktierenden Körper bei großen Eindrucktiefen eine, wenn auch geringe, Rolle spielt, kann dabei die gezeigte Kontaktlösung und die damit erhaltene Lösung des Stoßproblems im Allgemeinen (besonders für große Geschwindigkeiten) nicht ohne Weiteres für die Kollision elasto-plastischer Kugeln herangezogen werden.

Durch numerische Experimente lässt sich leicht zeigen, dass die Stoßzahl in diesem Modell fast ausschließlich von dem Verhältnis $v_{0} / v_{Y}^{*}(v)$ abhängt, wobei $v_{Y}^{*}$ die kritische Geschwindigkeit bezeichnet, die nötig ist, um in dem Modell plastische Deformationen zu erzeugen, und die durch die Beziehung 
Abb.5.17 Stoßzahl als Funktion der normierten Stoßgeschwindigkeit für den elasto-plastischen Normalstoß von Kugeln nach den unterschiedlichen Modellen. Die dünne Linie bezeichnet die asymptotische Näherung (5.106)

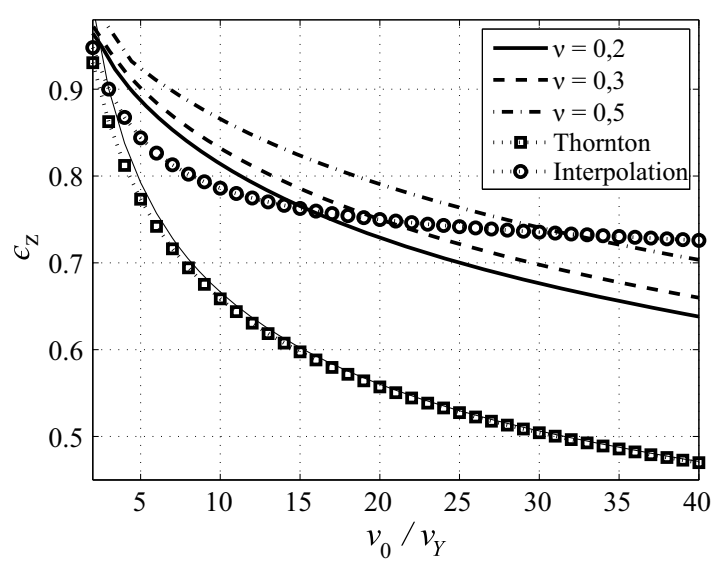

$$
\frac{v_{Y}^{*}(v)}{v_{Y}}=\left(\frac{5 C(v)}{8}\right)^{5 / 2}, \quad C(v):=1,295 \exp (0,736 v)
$$

aus der in Gl. (5.103) gegebenen Geschwindigkeit $v_{Y}$ bestimmt werden kann. Die explizite Abhängigkeit der Stoßzahl von den Parametern $\sigma_{Y} / \tilde{E}$ und $v$ ist sehr schwach. Von dem Radius oder der Masse der Kugel hängt die Stoßzahl überhaupt nicht ab.

Abb. 5.17 zeigt die unterschiedlichen Lösungen für die Stoßzahl als Funktion der auf $v_{Y}$ normierten Stoßgeschwindigkeit nach dem Modell von Thornton, dem Interpolationsmodell - mit $Q=5$ und $D=80$, siehe Gl.(3.282) - und dem Modell auf der Grundlage der analytischen Approximation von rigorosen FEM-Rechnungen (für $\sigma_{Y} / \tilde{E}=1 / 500$ sowie drei verschiedene Werte von $v^{15}$ ). Man erkennt, dass das Modell von Thornton eine deutlich kleinere Stoßzahl vorhersagt als die anderen Modelle. Dies wurde bereits im dritten Kapitel begründet. Die Kurve des Interpolationsmodells fällt auch zunächst steil ab, wird dann aber sogar flacher als die Vorhersage der FEM-basierten Rechnungen.

\subsubsection{Vergleich mit experimentellen Ergebnissen}

Es gibt in der Literatur mehrere experimentelle Studien zu der elasto-plastischen Kollision einer Stahlkugel auf eine massive Stahlplatte, z. B. von Lifshitz und Kolsky [45] oder Wong et al. [46]. Da in diesen Arbeiten allerdings beide Kontaktpartner die gleichen mechanischen Eigenschaften aufweisen - während das oben verwendete FEM-basierte Modell ausdrücklich für Indentierungsprobleme gedacht ist, bei denen die Platte eine deutlich geringere Festigkeit hat als die eindringende Kugel - soll an dieser Stelle für den Vergleich der theoretischen Vorhersagen mit experimentellen Ergebnissen die Arbeit von Kharaz und Gorham

\footnotetext{
${ }^{15}$ Dies beeinflusst hauptsächlich die Geschwindigkeit $v_{Y}^{*}$; die explizite Abhängigkeit der Stoßzahl von $v$ für ein konstantes $v_{Y}^{*}$ ist, wie gesagt, sehr schwach.
} 
Tab.5.2 Die für die theoretischen Vorhersagen verwendeten Materialkennwerte

\begin{tabular}{l|l|l|l|l}
\hline Material & E-Modul [GPa] & Poissonzahl & $\begin{array}{l}\text { Vickers-Härte } \\
{[\mathrm{GPa}]}\end{array}$ & $\begin{array}{l}\text { Streckgrenze } \\
{[\mathrm{MPa}]}\end{array}$ \\
\hline Aluminiumoxid & 360 & 0,23 & - & - \\
\hline Stahl & 206 & 0,29 & $3,09$ (nach $[47])$ & 1030 \\
\hline Aluminium & 70 & 0,345 & $1,14$ (nach $[47])$ & 380 \\
\hline
\end{tabular}

Abb.5.18 Stoßzahl als

Funktion der

Stoßgeschwindigkeit für den elasto-plastischen Normalstoß einer Aluminiumoxid-Kugel auf eine dicke Stahlplatte. Vergleich der verschiedenen theoretischen Vorhersagen mit experimentellen Ergebnissen von Kharaz und Gorham [47]

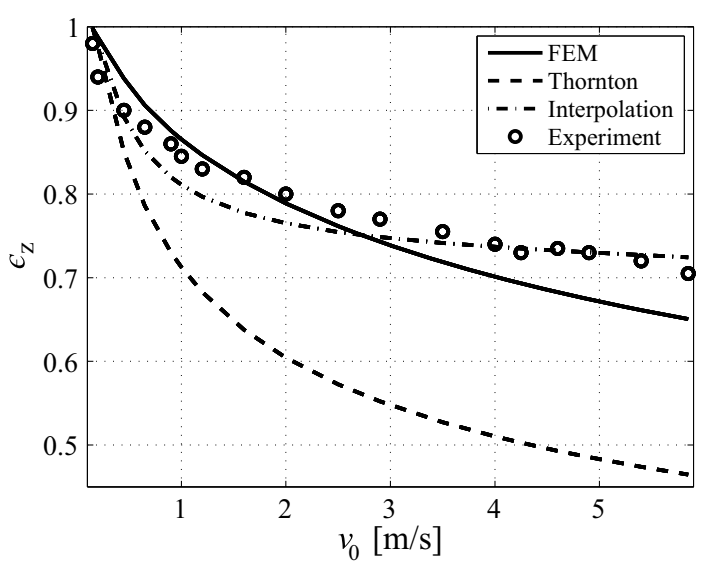

[47] herangezogen werden, in der Stöße von harten ${ }^{16}$ Aluminiumoxid-Kugeln auf eine massive Stahl-, bzw. Aluminiumplatte untersucht wurden. Die Kugeln hatten einen Radius von 2,5 mm und eine Masse von 0,25 g. Die relevanten Materialkennwerte sind in Tab.5.2 zusammengefasst; für keines der verwendeten theoretischen Modelle wurden die Parameter an die experimentellen Daten angepasst, um eine bessere Übereinstimmung zu erzielen.

Abb. 5.18 zeigt die experimentellen Resultate gemeinsam mit den Ergebnissen der vorgestellten Modelle für die Abhängigkeit der Stoßzahl als Funktion der Kollisionsgeschwindigkeit in dem elasto-plastischen Normalstoß einer Aluminiumoxid-Kugel auf eine Stahlplatte. Für eine Stoßgeschwindigkeit von $v_{0}=0,12 \mathrm{~m} / \mathrm{s}$ ist die Kollision praktisch ideal-elastisch. Bei etwa dem Fünfzigfachen dieser kritischen Geschwindigkeit fällt die Stoßzahl auf den Wert 0,7. Die Übereinstimmung mit den theoretischen Vorhersagen ist für das Interpolationsmodell und das FEM-basierte Modell gut; das Thorntonsche Modell sagt, bis auf einen kleinen Bereich in der Nähe des elastischen Grenzfalls, eine durchgängig deutlich zu kleine Stoßzahl voraus.

\footnotetext{
${ }^{16}$ Man muss eigentlich zwischen Härte, Festigkeit und Steifigkeit unterscheiden; in diesem Fall sind aber alle drei Varianten zutreffend.
} 
Abb.5.19 Stoßzahl als

Funktion der

Stoßgeschwindigkeit für den elasto-plastischen Normalstoß einer Aluminiumoxid-Kugel auf eine dicke

Aluminiumplatte. Vergleich der verschiedenen theoretischen

Vorhersagen mit

experimentellen Ergebnissen von Kharaz und Gorham [47]

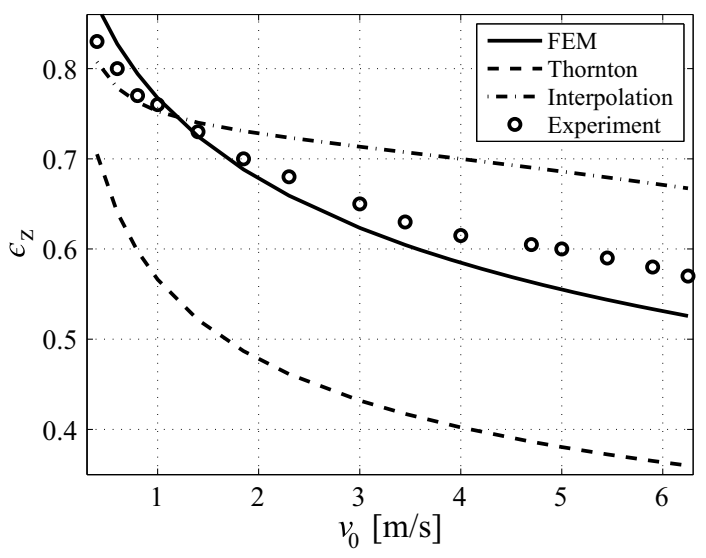

In Abb.5.19 sind die entsprechenden Kurven für eine Aluminiumplatte gezeigt. In der Theorie ergibt sich eine kritische Geschwindigkeit von ungefähr $0,05 \mathrm{~m} / \mathrm{s}$ zur Initiierung plastischer Verformungen. In diesem Geschwindigkeitsbereich wurden aber von den Autor*innen der experimentellen Studie leider keine Messungen durchgeführt. Die Übereinstimmung mit den Prognosen des FEM-basierten Modells ist wiederum gut; das Interpolationsmodell weicht für größere Geschwindigkeiten zunehmend nach oben von den Versuchsergebnissen ab, die Vorhersagefähigkeit des Thorntonschen Modells ist erneut unbefriedigend.

Zusammenfassend kann man bilanzieren, dass das Modell von Thornton nur für einen kleinen Bereich in der Nähe des elastischen Grenzfalls korrekte Ergebnisse liefert, für gröBere Geschwindigkeiten wird die Energiedissipation von dem Modell deutlich überschätzt. Das Interpolationsmodell liefert für kleine und mittlere Geschwindigkeiten gute Vorhersagen, für große Geschwindigkeiten (in der Größenordnung des Hundertfachen der kritischen Geschwindigkeit) wird die plastische Dissipation von dem Modell zunehmend deutlich unterschätzt. Auch das FEM-basierte Modell kann keine sehr gute Übereinstimmung mit den Versuchsergebnissen erreichen, was vermutlich darauf zurückzuführen ist, dass die Verfestigung des plastisch deformierten Mediums nicht ausreichend in dem zugrundeliegenden FEM-Modell berücksichtigt wurde [44].

\subsection{Elasto-Plastischer Normalstoß mit Adhäsion}

Wegen der Spannungskonzentration am Rand des adhäsiven Kontaktes spielt Plastizität in adhäsiven Stößen eine noch größere Rolle, als das schon bei nicht-adhäsiven Kollisionen der Fall ist. Nichtsdestotrotz ist das adhäsive Stoßproblem von elasto-plastischen Körpern weit entfernt von einer umfassenden, rigorosen Beschreibung, da bereits die zugrundeliegende 
Kontaktmechanik noch in großen Teilen unverstanden ist. Allerdings gibt es einige theoretische Ansätze, wie man die Aufgabe zumindest qualitativ behandeln kann.

Die erste Arbeit zu dem Thema stammt von Johnson und Pollock [9]. Diese nahmen an, dass die makroskopischen Kontaktgrößen während der Kollision immer noch den Relationen der JKR-Theorie genügen, wobei die Oberflächenenergie durch die plastische Deformation mit einem Faktor $k$ skaliert wird, der während der Kompressionsphase kleiner als Eins und während der Restitutionsphase größer als Eins ist. Die während des Stoßes dissipierte kinetische Energie und damit die Stoßzahl kann dann als Funktion der beiden Werte von $k$ bestimmt werden. Leider gaben Johnson \& Pollock keine Möglichkeit an, wie diese Werte aus den Parametern der Kollision ermittelbar sind; da sie den Einfluss der plastischen Deformation charakterisieren sollen, sind sie sicherlich selbst beispielsweise von der Stoßgeschwindigkeit stark abhängig.

Thornton und Ning [10] verwendeten ebenfalls die Kontaktlösung der JKR-Theorie und die Idee von Thornton, die Druckverteilung im Kontakt durch die Fließgrenze des Materials zu beschränken. Das entstehende Bewegungsgleichungssystem lösten sie numerisch und gaben außerdem eine analytische Lösung mithilfe eines einfachen Superpositionsmodells an, das auch von Kim und Dunn [48] verwendet wurde und das weiter unten kurz beschrieben ist. Allerdings wurde schon bei der Betrachtung des Problems ohne Adhäsion gezeigt, dass das Modell von Thornton den Kontakt nur in der Nähe des elastischen Grenzfalls zufriedenstellend beschreibt, da der Kontaktdruck im elasto-plastischen Bereich weiter wächst.

In einer vor Kurzem erschienenen Arbeit untersuchten Ghanbarzadeh et al. [49] das Problem mithilfe der Randelemente-Methode (boundary element method, BEM) für den elastischen Halbraum. Die Adhäsion berücksichtigten sie auf rigorose Weise durch ein mesh-abhängiges Spannungskriterium, das auf Pohrt und Popov [50] zurückgeht. Plastische Deformationen führten die Autor*innen dagegen nicht-rigoros ein, indem sie den Druck in einer BEM-Zelle, in Analogie zu dem Thorntonschen Modell, durch die Fließgrenze beschränkten. Die genannte Publikation war auch die erste, in der der Effekt der Rauigkeit der beteiligten Oberflächen auf das Stoßproblem systematisch untersucht wurde. Die Autor*innen stellten dabei fest, dass die Rauigkeit erst relevant wird, wenn sie von der gleichen Größenordnung wie die maximale Eindrucktiefe während der Kollision ist. Ansonsten kann man ohne Schwierigkeiten von makroskopisch glatten Oberflächen ausgehen.

\section{Einfache Superposition der Dissipationsmechanismen}

Ein einfacher qualitativer Ansatz zur Lösung des Problems besteht darin, die dissipierten Energien des elastischen adhäsiven und des elasto-plastischen nicht-adhäsiven Problems einfach zu addieren. Für die Stoßzahl im Fall mit Plastizität und Adhäsion erhält man dann

$$
\left(1-\epsilon_{z}^{2}\right)=\left(1-\epsilon_{\mathrm{adh}}^{2}\right)+\left(1-\epsilon_{\mathrm{pl}}^{2}\right) .
$$


Da die Stoßzahl des elastischen adhäsiven Problems in geschlossen analytischer Form durch Gl. (5.37) bestimmt ist, erhält man mit der Anfangsgeschwindigkeit $v_{0}$ und der in Gl. (5.38) gegebenen kritischen Geschwindigkeit $v_{c}$ des elastischen Problems den Ausdruck

$$
\epsilon_{z}=\sqrt{\epsilon_{\mathrm{pl}}^{2}-\frac{v_{c}^{2}}{v_{0}^{2}}}
$$

Zur Bestimmung von $\epsilon_{\mathrm{pl}}$ kann man dabei die im vorherigen Unterkapitel vorgestellten Modelle heranziehen.

\section{Interpolation im elasto-plastischen Bereich}

Adhäsion und Plastizität sind natürlich keine unabhängigen Phänomene, wie das die obige Superposition impliziert. Beispielsweise sind im adhäsiven Kontakt geringere Lasten zur Generierung von plastischer Deformation nötig; die Dissipation durch inelastische Verformungen wird also durch die Adhäsion verstärkt. Andererseits verändert sich durch die plastische Deformation das Profil der kontaktierenden Körper in der Umgebung des Kontaktes, was wiederum die adhäsive Wechselwirkung beeinflusst. Um diese gegenseitigen Einflüsse zumindest qualitativ zu erfassen, soll im Folgenden ein grobes analytisches Modell für den adhäsiven Kontakt elasto-plastischer Körper vorgestellt werden, das auf unterschiedliche, bisher in dem vorliegenden Buch geschilderte Konzepte zurückgreift, und das man auch zur Lösung des entsprechenden Stoßproblems heranziehen kann.

Das Modell beruht auf der Interpolation im elasto-plastischen Bereich zwischen der elastischen und der voll-plastischen Lösung, die sich schon im nicht-adhäsiven Fall als einfaches aber vergleichsweise robustes analytisches Verfahren bewährt hat.

Für die Kollision von Kugeln ist die elastische Lösung durch die JKR-Theorie gegeben. Normiert man alle Größen auf ihre Werte in der kritischen Konfiguration, bei der der Kontakt unter kraftgesteuerten Bedingungen seine Stabilität verliert ${ }^{17}$, sind die Zusammenhänge zwischen Eindrucktiefe, Kontaktradius und Normalkraft durch die Gl.(3.63) und (3.64) gegeben. Für die Poissonzahl $v=0,3$ behält die elastische Lösung in der Kompressionsphase ihre Gültigkeit, solange die Bedingung [51]

$$
-\frac{2 F_{z}}{3 \pi \Delta \gamma \tilde{R}}<\hat{F}_{Y}(\Phi)=2\left(0,111 \Phi-0,8236-\frac{0,0636}{\Phi}+\frac{0,0087}{\Phi^{2}}+\frac{1,2429}{\Phi^{3}}\right),
$$

mit dem in Gl.(3.293) eingeführten Adhäsionsparameter, erfüllt ist ${ }^{18}$. Die zugehörigen Werte des normierten Kontaktradius und der normierten Eindrucktiefe sind

\footnotetext{
${ }^{17}$ Alle so normierten Größen sind im Folgenden durch einen „Hut“ auf der entsprechenden Größe gekennzeichnet.

${ }^{18}$ Die plastische Deformation am Rand des Kontaktes wird an dieser Stelle vernachlässigt, da sie auf einen sehr kleinen Bereich beschränkt ist [51].
} 


$$
\begin{aligned}
& \hat{a}_{Y}=\left(1+\sqrt{1+\hat{F}_{Y}}\right)^{2 / 3}, \\
& \hat{d}_{Y}=3 \hat{a}_{Y}^{2}-4 \sqrt{\hat{a}_{Y}} .
\end{aligned}
$$

In Ermangelung entsprechender Untersuchungen sei angenommen, dass die voll-plastische Lösung - zumindest was die Zusammenhänge zwischen den makroskopischen Kontaktgrößen angeht - von der Adhäsion unbeeinflusst bleibt ${ }^{19}$. In normierten Größen ergibt sich dann

$$
-\hat{F}_{z}^{\mathrm{pl}}=\frac{\tilde{Q}}{\Phi} \hat{a}_{\mathrm{pl}}^{2}=\frac{2 \tilde{Q}}{3 \Phi} \hat{d}, \quad \hat{d}>\hat{d}_{0}:=\frac{\tilde{D}}{\Phi^{2}},
$$

mit $\tilde{Q} \approx 3,5$ und $\tilde{D} \approx 400$. Interpoliert man nun im elasto-plastischen Bereich mithilfe von Polynomen dritten Grades so, dass die Zusammenhänge zwischen den makroskopischen Größen an den Übergangsstellen stetig und stetig differenzierbar sind, erhält man für die normierte Normalkraft im elasto-plastischen Bereich

$$
-\hat{F}_{z}^{\mathrm{ep}}=\hat{F}_{Y}+C_{1}\left(\hat{d}-\hat{d}_{Y}\right)+C_{2}\left(\hat{d}-\hat{d}_{Y}\right)^{2}+C_{3}\left(\hat{d}-\hat{d}_{Y}\right)^{3},
$$

mit

$$
\begin{aligned}
& C_{1}=\frac{3 \hat{a}_{Y}^{2}-3 \hat{a}_{Y}^{1 / 2}}{6 \hat{a}_{Y}-2 \hat{a}_{Y}^{-1 / 2}}, \quad C_{3}=\frac{2\left(\hat{F}_{Y}-\hat{F}_{0}\right)+\hat{F}_{0}^{\prime}\left(\hat{d}_{0}-\hat{d}_{Y}\right)+C_{1}\left(\hat{d}_{0}-\hat{d}_{Y}\right)}{\left(\hat{d}_{0}-\hat{d}_{Y}\right)^{3}}, \\
& C_{2}=\frac{3\left(\hat{F}_{0}-\hat{F}_{Y}\right)-\hat{F}_{0}^{\prime}\left(\hat{d}_{0}-\hat{d}_{Y}\right)-2 C_{1}\left(\hat{d}_{0}-\hat{d}_{Y}\right)}{\left(\hat{d}_{0}-\hat{d}_{Y}\right)^{2}}, \quad \hat{F}_{0}=\frac{2 \tilde{Q} \tilde{D}}{3 \Phi^{3}}, \quad \hat{F}_{0}^{\prime}=\frac{2 \tilde{Q}}{3 \Phi},
\end{aligned}
$$

und für den normierten Kontaktradius

$$
\hat{a}_{\mathrm{ep}}=\hat{a}_{Y}+C_{4}\left(\hat{d}-\hat{d}_{Y}\right)+C_{5}\left(\hat{d}-\hat{d}_{Y}\right)^{2}+C_{6}\left(\hat{d}-\hat{d}_{Y}\right)^{3},
$$

mit

\footnotetext{
${ }^{19}$ Dies kann man so interpretieren, dass die effektive Oberflächenenergie bei voll-plastischer Kompression verschwindet, siehe das Modell von Johnson und Pollock [9].
} 


$$
\begin{aligned}
& C_{4}=\frac{1}{6 \hat{a}_{Y}-2 \hat{a}_{Y}^{-1 / 2}}, \quad C_{6}=\frac{2\left(\hat{a}_{Y}-\hat{a}_{0}\right)+\hat{a}_{0}^{\prime}\left(\hat{d}_{0}-\hat{d}_{Y}\right)+C_{4}\left(\hat{d}_{0}-\hat{d}_{Y}\right)}{\left(\hat{d}_{0}-\hat{d}_{Y}\right)^{3}}, \\
& C_{5}=\frac{3\left(\hat{a}_{0}-\hat{a}_{Y}\right)-\hat{a}_{0}^{\prime}\left(\hat{d}_{0}-\hat{d}_{Y}\right)-2 C_{4}\left(\hat{d}_{0}-\hat{d}_{Y}\right)}{\left(\hat{d}_{0}-\hat{d}_{Y}\right)^{2}}, \quad \hat{a}_{0}=\sqrt{\frac{2 \tilde{D}}{3 \Phi^{2}}}, \quad \hat{a}_{0}^{\prime}=\sqrt{\frac{\Phi^{2}}{6 \tilde{D}}} .
\end{aligned}
$$

Die Restitution wird wie im nicht-adhäsiven Fall als elastischer Prozess mit einem durch die vorhergehende plastische Deformation veränderten Krümmungsradius aufgefasst. Die Kontaktlösung ergibt sich dann wiederum aus der JKR-Theorie zu

$$
\begin{aligned}
d-d_{\text {res }} & =\frac{a^{2}}{R_{p}}-\sqrt{\frac{2 \pi a \Delta \gamma}{\tilde{E}},} \\
F_{z} & =-\frac{4}{3} \tilde{E} \frac{a^{3}}{\tilde{R}_{p}}+\sqrt{8 \pi a^{3} \Delta \gamma \tilde{E}},
\end{aligned}
$$

wobei wegen der Stetigkeit aller Größen am Umkehrpunkt der neue Krümmungsradius $R_{p}$ und die Resteindrucktiefe $d_{\text {res }}$ durch

$$
\begin{gathered}
R_{p}=\frac{4 \tilde{E} a_{\max }^{3}}{3\left|F_{z, \max }\right|+\sqrt{8 \pi a_{\max }^{3} \Delta \gamma \tilde{E}}}, \\
d_{\mathrm{res}}=d_{\max }-\frac{a_{\max }^{2}}{R_{p}}+\sqrt{\frac{2 \pi a_{\max } \Delta \gamma}{\tilde{E}}} .
\end{gathered}
$$

gegeben sind. Der Stoß ist beendet, wenn der Kontakt seine Stabilität verliert, d.h. wenn

$$
d-d_{\mathrm{res}}=d_{c}^{\mathrm{WS}}=-\frac{3}{4}\left(\frac{\pi^{2} \Delta \gamma^{2} \tilde{R}_{p}}{\tilde{E}^{2}}\right)^{1 / 3} .
$$

Die Stoßzahl in diesem Modell hängt nur von dem Verhältnis $v_{0} / v_{c}$ sowie dem Parameter $\Phi$ ab. Abb.5.20 zeigt die Stoßzahl als Funktion der normierten Stoßgeschwindigkeit für verschiedene Werte von $\Phi$ gemeinsam mit der entsprechenden Lösung durch die einfache Superposition der Dissipationsmechanismen. Die nicht-adhäsive elasto-plastische Stoßzahl wurde dabei für letztere ebenfalls mithilfe des Interpolationsmodells gewonnen, um einfacher vergleichen zu können. Das dafür notwendige Verhältnis $v_{c} / v_{Y}$ kann man dabei durch die Beziehung

$$
\frac{v_{c}^{2}}{v_{Y}^{2}} \approx 0,37 \Phi^{5}
$$

aus dem Adhäsionsparameter $\Phi$ bestimmen. 
Abb.5.20 Stoßzahl als
Funktion der normierten
Stoßgeschwindigkeit für den
elasto-plastischen Normalstoß
von Kugeln mit Adhäsion nach
den beiden Modellen. Die
Kreise bezeichnen die Lösung
nach dem Interpolationsmodell.
Die Kreuze bezeichnen das
Ergebnis durch einfache
Superposition der
Dissipationsmechanismen. Die
dünne Linie beschreibt die
elastische Lösung

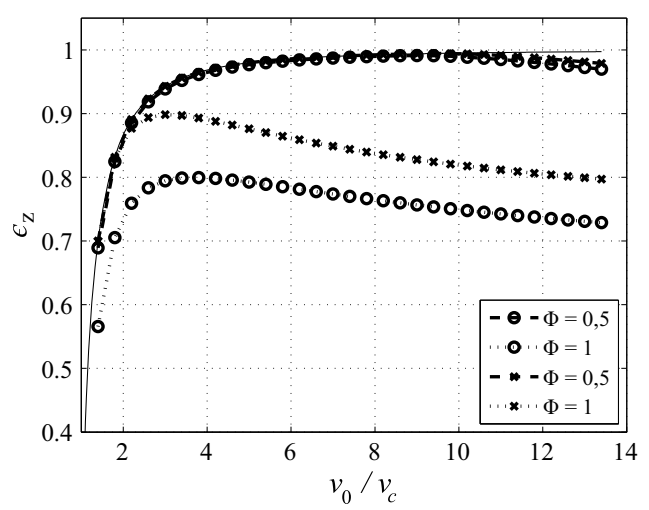

Man erkennt, dass die Energiedissipation von der einfachen Superposition für den Fall $\Phi=1$ geringer geschätzt wird als von dem adhäsiven Interpolationsmodell. Dies liegt daran, dass durch die einfache Superposition nicht berücksichtigt wird, dass das lokale Fließen wegen der Adhäsion früher beginnt. Für $\Phi=0,5$ gibt es dagegen bereits kaum einen Unterschied zwischen den beiden Modellen. Das ist nicht überraschend, da für diesen Wert des Adhäsionsparameters die kritische Kraft, um Fließen zu initiieren, nur geringfügig kleiner ist als im nicht-adhäsiven Fall (siehe Abb. 3.19).

\subsection{Zusammenfassung}

Dieses Kapitel widmete sich langsamen (d.h. quasistatischen) zentrischen Normalstößen axialsymmetrischer Körper. Zur Lösung des Stoßproblems wurde mithilfe der im dritten Kapitel dargelegten Grundlagen des Normalkontaktproblems die Bewegungsgleichung für die Indentierungstiefe bestimmt und analytisch oder numerisch integriert.

Im Fall der elastischen Kollision ohne Adhäsion kann man die Bewegungsgleichung vollständig analytisch lösen. Die Eindrucktiefe als Funktion der Zeit hängt in normierten Größen dabei nur sehr schwach von der Form der zusammenstoßenden Körper ab und kann daher gut durch den sinus-förmigen Verlauf des vollständig linearen Kontaktes angenähert werden. Für Funktionale Gradientenmedien ist die normierte Bahnkurve bestimmbar, indem ein äquivalentes homogenes Problem mit einer entsprechend angepassten Profilform gelöst wird. Der Maximaldruck während des Stoßes ist für den parabolischen Kontakt durch eine passend gewählte elastische Gradierung deutlich reduzierbar.

Bei der elastischen Kollision mit Adhäsion wird gegen die Wirkung der Adhäsion eine Energiemenge dissipiert, die von den elastischen, geometrischen und adhäsiven Eigenschaften des Systems abhängt, aber nicht von der Stoßgeschwindigkeit. Bei ausreichend kleinen Geschwindigkeiten kann es daher dazu kommen, dass sich die zusammenstoßenden Körper nicht wieder voneinander lösen. Eine Untersuchung des Einflusses der Reichweite der 
Adhäsion (im Rahmen der Maugis-Theorie) zeigte dabei, dass der Energie-Verlust durch die JKR-Theorie auch für große Werte des Tabor-Parameters deutlich überschätzt wird.

In der Kollision viskoelastischer Körper hängt die Stoßzahl nur schwach von der Profilform und der konkreten viskoelastischen Rheologie ab. Die dominierende Größe ist das Verhältnis des Verlust- und Speichermoduls bei der charakteristischen Zeitskala des Stoßes, d. h. der Stoßdauer. Als gute Näherungslösung des Stoßproblems kann daher die analytische Lösung für den Stoß eines zylindrischen Flachstempels auf ein Kelvin-Voigt-Medium dienen. Das Modell von Kuwabara \& Kono für die Kollision viskoelastischer Kugeln ist zwar aus kontaktmechanischer Sicht leicht inkorrekt, liefert aber praktisch die gleichen Ergebnisse wie ein kontaktmechanisch rigoroses Modell und ist dabei einfacher zu implementieren und handzuhaben. Die Kompressibilität des viskoelastischen Materials ist von geringer Bedeutung für das Kollisionsverhalten; dabei führt die Annahme der Inkompressibilität in der Regel zu einer Überschätzung der Dissipation und damit zu einer Unterschätzung der Stoßzahl.

Für den elasto-plastischen Normalstoß ohne Adhäsion hängt die Stoßzahl praktisch nur von dem Verhältnis der Stoßgeschwindigkeit zur kritischen Geschwindigkeit ab, die nötig ist, um bei der Kollision relevante plastische Deformationen zu erzeugen. Bei der theoretischen Modellierung des Stoßproblems bestehen dabei immer noch Defizite, beispielsweise in der Berücksichtigung der Verfestigung.

Der elasto-plastische Stoß mit Adhäsion ist noch in großen Teilen unverstanden. Es wurde aber gezeigt, dass eine einfache Superposition der beiden Dissipationsmechanismen (Adhäsion und plastische Deformation) die Gesamtdissipation unterschätzt, da die einfache Superposition die relevanten Wechselwirkungen von Adhäsion und Plastizität ignoriert.

\section{Literatur}

1. Hertz, H. (1882). Über die Berührung fester elastischer Körper. Journal für die reine und angewandte Mathematik, 92(1882), 156-171.

2. Eason, G. (1996). The displacements produced in an elastic half-space by a suddenly applied surface force. IMA Journal of Applied Mathematics, 2(4), 299-326.

3. Love, A. E. H. (1944). A treatise on the mathematical theory of elasticity (4. Aufl.). New York: Dover Publications.

4. Hunter, S. C. (1957). Energy absorbed by elastic waves during impact. Journal of the Mechanics and Physics of Solids, 5(3), 162-171.

5. Goldsmith, W. (1960). Impact: The theory and physical behaviour of colliding solids. London: Edward Arnold Publishers Ltd.

6. Deresiewicz, H. (1968). A note on Hertz's theory of impact. Acta Mechanica, 6, 110-112.

7. Graham, G. A. C. (1973). A contribution to Hertz's theory of elastic impact. International Journal of Engineering Science, 11(4), 409-413.

8. Willert, E., \& Popov, V. L. The oblique impact of a rigid sphere on a power-law graded elastic halfspace. Mechanics of Materials, 109, 82-89.

9. Johnson, K. L., \& Pollock, H. M. (1994) The role of adhesion in the impact of elastic spheres. Journal of Adhesion Science and Technology, 8(11), 1323-1332. 
10. Thornton, C., \& Ning, Z. (1998). A theoretical model for the stick/bounce behaviour of adhesive elasticplastic spheres. Powder Technology, 99(2), 154-162.

11. Willert, E., Lyashenko, I. A., \& Popov, V. L. (2013). Influence of the Tabor parameter on the adhesive normal impact of spheres in Maugis-Dugdale approximation. Computational Particle Mechanics, 5(3), 313-318.

12. Ciavarella, M., Greenwood, J. A., \& Barber, J. R. (2017). Effect of Tabor parameter on hysteresis losses during adhesive contact. Journal of the Mechanics and Physics of Solids, 98, 236-244.

13. Wu, J. J. (2010). The jump-to-contact distance in atomic force microscopy measurement. The Journal of Adhesion, 86(11), 1071-1085.

14. Willert, E., \& Popov, V. L. (2017). Adhesive tangential impact without slip of a rigid sphere and a powerlaw graded elastic half-space. ZAMM Zeitschrift für Angewandte Mathematik und Mechanik, 97(7), 872-878.

15. Butcher, E. A., \& Segalman, D. J. (2000). Characterizing Damping and Restitution in Compliant Impacts via Modified K-V and Higher-Order Linear Viscoelastic Models. Journal of AppliedMechanics, 67(4), 831-834.

16. Schwager, T., \& Pöschel, T. (2007). Coefficient of restitution and linear-dashpot model revisited. Granular Matter, 9, 465-469.

17. Argatov, I. I. (2013). Mathematical modeling of linear viscoelastic impact: Application to drop impact testing of articular cartilage. Tribology International, 63, 213-225.

18. Kuwabara, G., \& Kono, K. (1987). Restitution coefficient in a collision between two spheres. Japanese Journal of Applied Physics, 26(8), 1230-1233.

19. Brilliantov, N. V., Spahn, F., Hertzsch, J. M., \& Pöschel, T. (1996). Model for collisions in granular gases. Physical Review, E 53(5), 5382-5392.

20. Ramírez, R., Pöschel, T., Brilliantov, N. V., \& Schwager, T. (1999). Coefficient of restitution of colliding viscoelastic spheres. Physical Review, E 60(4), 4465-4472.

21. Schwager, T., \& Pöschel, T. (2008). Coefficient of restitution for viscoelastic spheres: The effect of delayed recovery. Physical Review, E 78(8), 051304. https://doi.org/10.1103/PhysRevE.78. 051304.

22. Müller, P., \& Pöschel, T. (2011). Collision of viscoelastic spheres: Compact expressions for the coefficient of normal restitution. Physical Review, E 84(2), 021302. https://doi.org/10.1103/ PhysRevE.84.021302.

23. Pao, Y. H. (1955). Extension of the Hertz theory of impact to the viscoelastic case. Journal of Applied Physics, 26(9), 1083-1088.

24. Willert, E., Kusche, S., \& Popov, V. L. (2017). The influence if viscoelasticity on velocitydependent restitutions in the oblique impact of spheres. Facta Universitatis, Series Mechanical Engineering, 15(2), 269-284.

25. Lee, J., \& Herrmann, H. J. (1993). Angle of repose and angle of marginal stability: Molecular dynamics of granular particles. Journal of Physics A: General Physics, 26(2), 373-383.

26. Ray, S., Kempe, T., \& Fröhlich, J. (2015). Efficient modelling of particle collisions using a non-linear viscoelastic contact force. International Journal of Multiphase Flow, 76, 101-110.

27. Hunt, K. H., \& Crossley, F. R. E. (1975). Coefficient of restitution interpreted as damping in vibroimpact. Journal of AppliedMechanics, 42(2), 440-445.

28. Tsuji, Y., Tanaka, T., \& Ishida, T. (1992). Lagrangian numerical simulation of plug flow of cohesionless particles in a horizontal pipe. Powder Technology, 71(3), 239-250.

29. Van Zeebroeck, M. et al. (2003). Determination of the dynamical behaviour of biological materials during impact using a pendulum device. Journal of Sound and Vibration, 266(3), 465-480.

30. Kusche, S. (2016). Simulation von Kontaktproblemen bei linearem viskoelastischem Materialverhalten. Diss. Technische Universität Berlin, 2016. 
31. Brilliantov, N. V., Pimenova, A. V., \& Goldobin, D. S. (2015). A dissipative force between colliding viscoelastic bodies: Rigorous approach. EPL Europhysics Letters, 109(1), 14005. https:// doi.org/10.1209/0295-5075/109/14005.

32. Willert, E., Leroy, J.-E., Satora, M., \& Scholtyssek, Y. (2018). The influence of compressibility on the restitution coefficient for viscoelastic spheres in low-velocity normal impacts. https://arxiv. org/abs/1806.06540.

33. Tschoegl, N. W., Knauss, W. G., \& Emri, I. (2002). Poisson's ratio in linear viscoelasticity - A critical review. Mechanics of Time-Dependent Materials, 6(1), 3-51.

34. Yee, A. F., \& Takemori, M. T. (1982). Dynamic bulk and shear relaxation in glassy polymers. I. experimental techniques and results on PMMA. Journal of Polymer Science, Part B: Polymer Physics, 20(2), 205-224.

35. Stronge, W. J. (2000). Impact Mechanics. Cambridge: Cambridge University Press.

36. Weir, G., \& Tallon, S. (2005). The coefficient of restitution for normal incident, low velocity particle impacts. Chemical Engineering Science, 60(13), 3637-3647.

37. Li, L. Y., Thornton, C., \& Wu, C. Y. (2000). Impact behaviour of elastoplastic spheres with a rigid wall. Proceedings of the Institution of Mechanical Engineers, Part C: Journal of Mechanical Engineering Science, 214(8), 1107-114.

38. Wu, C. Y., Li, L. Y., \& Thornton, C. (2003). Rebound behaviour of spheres for plastic impacts. International Journal of Impact Engineering, 28(9), 929-946.

39. Wu, C. Y., \& Li, L. Y., Thornton, C. (2005). Energy dissipation during normal impact of elastic and elasticplastic spheres. International Journal of Impact Engineering, 32(1-4), 593-604.

40. Thornton, C., Cummins, S. J., \& Cleary, P. W. (2013). An investigation of the comparative behaviour of alternative contact force models during inelastic collisions. Powder Technology, 233, 30-46.

41. Popov, V. L., Starčević, J., \& Willert, E. (2019). Materialtheorie: Vorlesungsskript Sommersemester 2019. Berlin: Technische Universität Berlin.

42. Lyashenko, I. A., \& Popov, V. L. (2018). Dynamic model of elastoplastic normal collision of spherical particles under nonlocal plasticity. Physics of the Solid State, 60(3), 566-570.

43. Thornton, C. (1997). Coefficient of restitution for collinear collisions of elastic-perfectly plastic spheres. Journal of Applied Mechanics, 64(2), 383-386.

44. Jackson, R. L., Green, I., \& Marghitu, D. B. (2010). Predicting the coefficient of restitution of impacting elastic-perfectly plastic spheres. Nonlinear Dynamics, 60(3), 217-229.

45. Lifshitz, J. M., \& Kolsky, H. (1964). Some experiments on anelastic rebound. Journal of the Mechanics and Physics of Solids, 12(1), 35-43.

46. Wong, C. X., Daniel, M. C., \& Rongong, J. A. (2009). Energy dissipation prediction of particle dampers. Journal of Sound and Vibration, 319(1-2), 91-118.

47. Kharaz, A. H., \& Gorham, D. A. (2000). A study of the restitution coefficient in elastic-plastic impact. Philosophical Magazine Letters, 80(8), 549-559.

48. Kim, O. V., \& Dunn, P. F. (2007). A microsphere-surface impact model for implementation in computational fluid dynamics. Aerosol Science, 38(5), 532-549.

49. Ghanbarzadeh, A., Hassanpour, A., \& Neville, A. (2019). A numerical model for calculation of the restitution coefficient of elastic-perfectly plastic and adhesive bodies with rough surfaces. Powder Technology, 345, 203-212.

50. Pohrt, R., \& Popov, V. L. (2015). Adhesive contact simulation of elastic solids using local mesh-dependent detachment criterion in boundary elements method. Facta niversitatis, Series Mechanical Engineering, 13(1), 3-10.

51. Wu, Y. C., \& Adams, G. G. (2008). Plastic yield conditions for adhesive contacts between a rigid sphere and an elastic half-space. Journal of Tribology, 131(1), 011403. https://doi.org/10.1115/ 1.3002329 . 
Open Access Dieses Kapitel wird unter der Creative Commons Namensnennung 4.0 International Lizenz (http://creativecommons.org/licenses/by/4.0/deed.de) veröffentlicht, welche die Nutzung, Vervielfältigung, Bearbeitung, Verbreitung und Wiedergabe in jeglichem Medium und Format erlaubt, sofern Sie den/die ursprünglichen Autor(en) und die Quelle ordnungsgemäß nennen, einen Link zur Creative Commons Lizenz beifügen und angeben, ob Änderungen vorgenommen wurden.

Die in diesem Kapitel enthaltenen Bilder und sonstiges Drittmaterial unterliegen ebenfalls der genannten Creative Commons Lizenz, sofern sich aus der Abbildungslegende nichts anderes ergibt. Sofern das betreffende Material nicht unter der genannten Creative Commons Lizenz steht und die betreffende Handlung nicht nach gesetzlichen Vorschriften erlaubt ist, ist für die oben aufgeführten Weiterverwendungen des Materials die Einwilligung des jeweiligen Rechteinhabers einzuholen. 